\title{
Néandertal et le feu au Paléolithique moyen ancien. Tour d'horizon des traces de son utilisation dans le Nord de la France
}

\author{
Neanderthal and fire during Early Middle Palaeolithic. \\ Overview of its use traces in Northern France \\ David Hérisson $^{\mathrm{a}, *}$, Jean-Luc Locht ${ }^{\mathrm{b}}$, Patrick Auguste ${ }^{\mathrm{c}}$, \\ Alain Tuffreau ${ }^{\mathrm{d}}$ \\ a Laboratoire Halma-Ipel, UMR8164 du CNRS, université Lille 3, Pont de Bois, BP 60149, \\ 59653 Villeneuve-d'Ascq cedex, France \\ ${ }^{\mathrm{b}}$ INRAP Nord-Picardie, 518, rue Saint-Fuscien, 80000 Amiens, France \\ ${ }^{\mathrm{c}}$ UMR8217 du CNRS, université Lille 1, laboratoire géosystèmes, bâtiment SN5, \\ 59655 Villeneuve-d'Ascq, France \\ ${ }^{\mathrm{d}}$ Laboratoire Halma-Ipel, UMR8164 du CNRS, université Lille 1, UFR de géographie et d'aménagement, \\ avenue Paul-Langevin, 59655 Villeneuve-d'Ascq, France
}

Disponible sur Internet le 15 novembre 2013

\section{Résumé}

Cet article a pour but de dresser un bilan des traces d'utilisation du feu par les premiers Néandertaliens du Nord de la France, durant la seconde partie du Saalien (SIM 8 à 6). Ce tour d'horizon rappelle la rareté des témoignages de feu durant la phase ancienne du Paléolithique moyen (300-130 ka BP) à l'échelle de l'Europe du Nord-Ouest. Pour le Nord de la France, seuls les gisements de Biache-Saint-Vaast et Therdonne présentent des vestiges de combustion. À Biache-Saint-Vaast, ce n'est pas moins de six niveaux qui présentent des indices de combustion : silex et restes fauniques brûlés et parfois charbons de bois. À Therdonne, en plus de nombreux silex et quelques rares restes fauniques brûlés, ont été mis au jour lors de la fouille du niveau N3 plusieurs zones riches en résidus organiques et micro-charbons de bois. L'ensemble des données recueillies concernant les témoins de combustion à Biache-Saint-Vaast et Therdonne est compilé, analysé et interprété. Cette démarche débouche sur l'établissement de l'utilisation du feu ou son absence dans les occupations saaliennes des Néandertaliens de France septentrionale et à une discussion concernant

\footnotetext{
* Auteur correspondant. 16, rue Morelle 80290 Namps-Au-Mont, France.

Adressese-mail: davidherisson@yahoo.fr, david.herisson@inrap.fr (D. Hérisson). 
les modalités de son utilisation notamment à Therdonne. En conclusion est brièvement discuté le statut du feu dans les premières occupations néandertaliennes et ses implications.

(C) 2013 Elsevier Masson SAS. Tous droits réservés.

Mots clés : Néandertal ; Feu ; Foyer ; Paléolithique moyen ; Saalien ; Nord de la France ; Biache-Saint-Vaast ; Therdonne

\begin{abstract}
This article aims at drawing up balance sheet of remains of fire use by the first Neanderthals of Northern France, during the second part of the Saalian (MIS 8 to 6). This overview reminds us the rarity of fire testimonies during Early Middle Palaeolithic (300-130 ky BP) on the scale of North-Western Europe. For Northern France, only the sites of Biache-Saint-Vaast and Therdonne present remains of combustion. At Biache-Saint-Vaast, it is not less than six levels, which present clues of combustion: burnt flint and faunal remains and sometimes charcoals. At Therdonne, besides burnt numerous flint and some rare faunal remains were brought to light during the excavation of level N3 several rich zones in organic residues and microcharcoals. All the datas collected concerning the clues of combustion at Biache-Saint-Vaast and Therdonne is compiled, analyzed and interpreted. This approach permits to establish the fire use or its absence in saalian occupations of Neanderthals of Northern France and to discuss modalities of its use, particularly at Therdonne. To conclude, fire status and its implications in first Neanderthals occupations are briefly discussed.
\end{abstract}

(C) 2013 Elsevier Masson SAS. All rights reserved.

Keywords: Neanderthal; Fire; Hearth; Middle Palaeolithic; Saalian; Northern France; Biache-Saint-Vaast; Therdonne

\title{
1. Introduction
}

L'usage du feu par les hommes préhistoriques est un thème récurrent en préhistoire (Black, 1931 ; Bordes, 1958 ; Broca, 1870 ; Clark et Harris, 1985 ; James, 1989 ; March et al., 1989 ; March et al., 2006 ; Meignen et al., 2000 ; Mellars, 1976 ; Oakley, 1955 ; Olive et Taborin, 1989 ; Perlès, 1977 ; Roebroeks et Villa, 2011 ; Rowlett, 2000 ; Schiegl et al., 1996 ; Théry-Parisot, 2001 ; Théry-Parisot et al., 2010). Le débat vivant et passionnel se porte sur les premières traces de contrôle du feu par les hominidés comme sur la diffusion de ce savoir-faire. Concernant les traces préliminaires de maîtrise du feu, les témoignages les plus anciens de foyer au-delà des 500000 ans restent discutés (Meignen et al., 2000 ; Rolland, 2004, p. 253-259). Actuellement, un consensus se dégage autour d'une utilisation certaine du feu aux environs de 400000 ans, maîtrise attribuable à Homo erectus ou à ses descendants eurasiatiques. Postérieurement à 400000 ans, certains perçoivent une généralisation de l'emploi du feu dans les gisements européens, perceptible avant l'Eémien (Roebroeks et Villa, 2011 ; Rolland, 2004). Ce constat est à tempérer pour d'autres qui considèrent que le nombre d'occurrences présentant des traces de combustion entre 400000 et 130000 ans est proportionnellement croissant au nombre d'occupations connues (Sandgathe et al., 2011). Quoi qu'il en soit, rarissimes sont les foyers pléistocènes parfaitement préservés ayant subi les affres du temps. La majorité des témoignages de l'utilisation du feu sur les gisements pléistocènes sont très altérés et épars. Dans ces cas de figure, seule la convergence de plusieurs indices est nécessaire pour attester de l'utilisation de feu (Roebroeks et Villa, 2011). Le présent article propose d'explorer la relation Homme-feu durant une période définie, la seconde partie du Saalien - SIM 8 à 6, soit entre 300 et 130 ka BP (Lisiecki et Raymo, 2005) -, phase durant laquelle l'emploi habituel du feu se généraliserait (Roebroeks et 


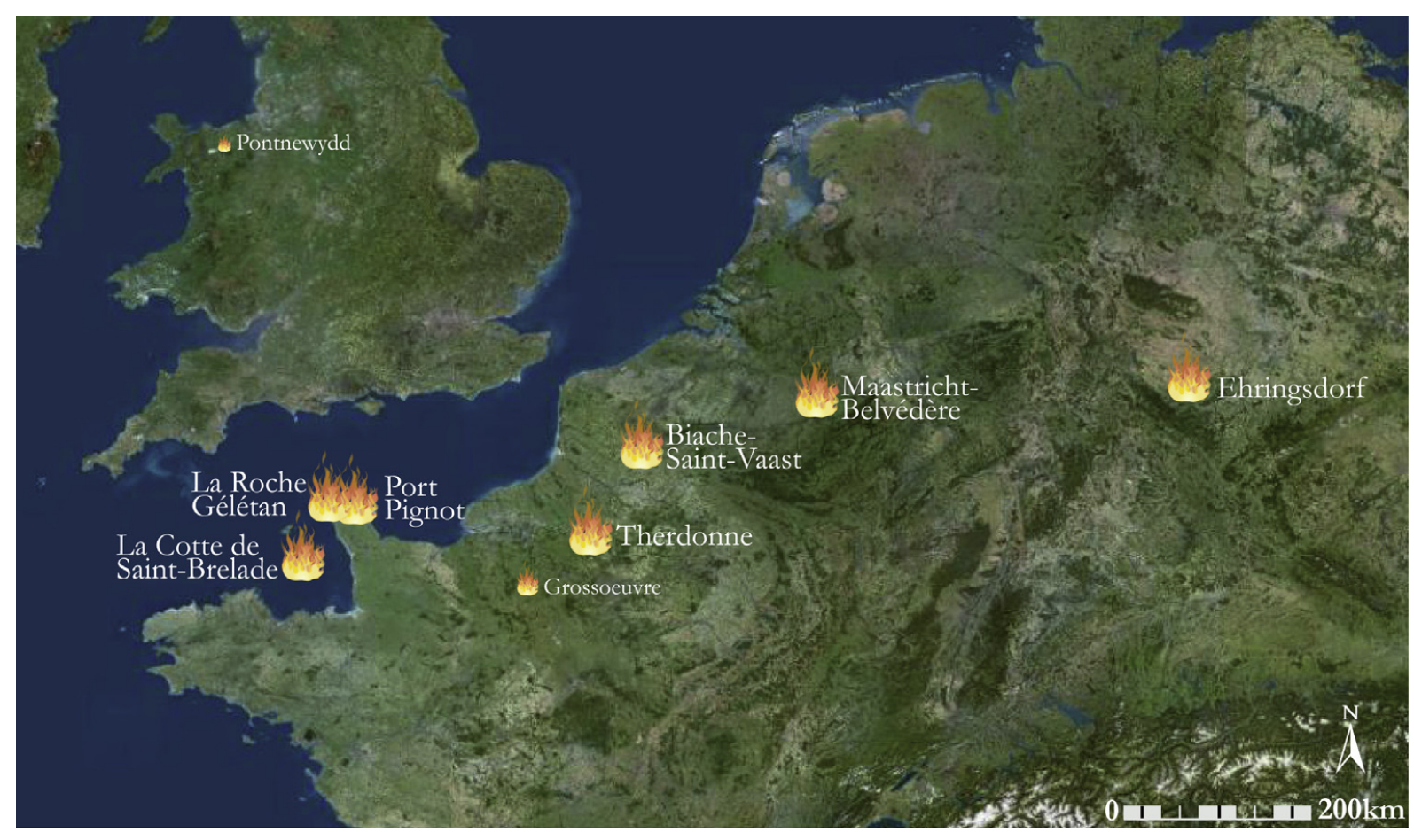

Fig. 1. Gisements saaliens (SIM 8 à 6 ; 300 à 130 ka BP) du Nord-Ouest de l'Europe possédant des traces de feu. La taille des foyers est proportionnelle à la fiabilité des indices attestant de l'utilisation du feu sur le site.

Saalian sites (MIS 8 to 6; 300 to $130 \mathrm{ky}$ BP) of North-western Europe with fire traces. The hearth size is proportional to the reliability of clues attesting fire use on the site.

Villa, 2011 ; Rolland, 2004, p. 253-259). Cette étude se penche sur un espace géographique particulier qu'est le Nord de la France, au sens de Tuffreau (2012) (p. 36) et qui s'insère dans l'aire européenne du Nord-Ouest, considérée comme des latitudes septentrionales (au-delà du $49^{\mathrm{e}}$ parallèle nord), hostiles pour l'Homme durant les phases pléniglaciaires. À l'échelle de l'Europe du Nord-Ouest, seuls neuf gisements recèlent des traces d'utilisation de feu (Fig. 1 et 2). Deux de ces neuf gisements, Biache-Saint-Vaast et Therdonne, se situent dans le Nord de la France. Le présent article se focalisera sur ces deux sites, seuls témoins de l'utilisation du feu par les premiers Néandertaliens durant le Saalien pour cet espace. Quelles traces de combustion subsistent dans les différentes occupations saaliennes fouillées à Biache-Saint-Vaast et Therdonne ? Quels éléments recèlent ces occupations quant à l'usage du feu au Saalien par les Néandertaliens septentrionaux ? Cette exploration thématique et inédite restitue des témoignages rares et peu documentés jusqu'à présent à travers les quelques pages qui suivent.

\section{Contexte des occupations saaliennes de Biache-Saint-Vaast et de Therdonne}

L'histoire du gisement de Biache-Saint-Vaast (Pas-de-Calais, Fig. 1) débuta au printemps 1976 par une découverte fortuite lors de l'agrandissement d'un bâtiment de l'usine sidérurgique Usinor (Tuffreau et Sommé, 1988a, p. 15). Une opération de sauvetage fut montée sous la direction d'A. Tuffreau, aidé de J.-F. Piningre et permit de fouiller quelques $340 \mathrm{~m}^{2}$ pour le niveau IIbase et la moitié pour le niveau IIA-II $\alpha$. Un sondage indiqua que le potentiel du gisement s'étendait à l'est et au sud-est de l'intervention de 1976. Cet élément déclencha une série de campagnes de fouilles programmées de 1977 à 1982. Ces travaux s'effectuèrent sur deux secteurs différents, appelés Chantier Nord et Chantier Sud. Le Chantier Nord livra la même séquence stratigraphique que le chantier de 1976 sur $1000 \mathrm{~m}^{2}$. Fouillé sur $500 \mathrm{~m}^{2}$, la stratigraphie 


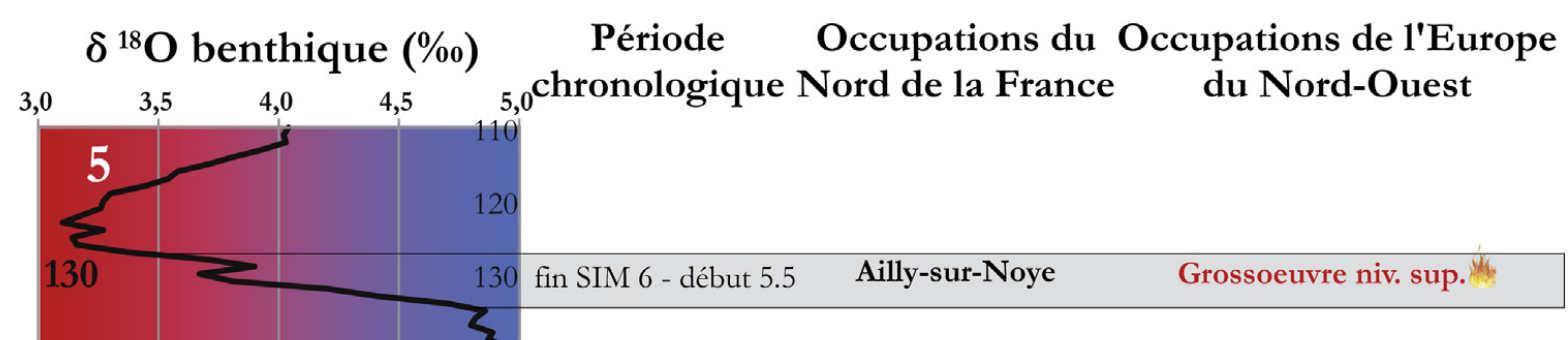

\Plachy-Buyon N1 La Roche Gélétan niv. Frais

Plachy-Buyon N2
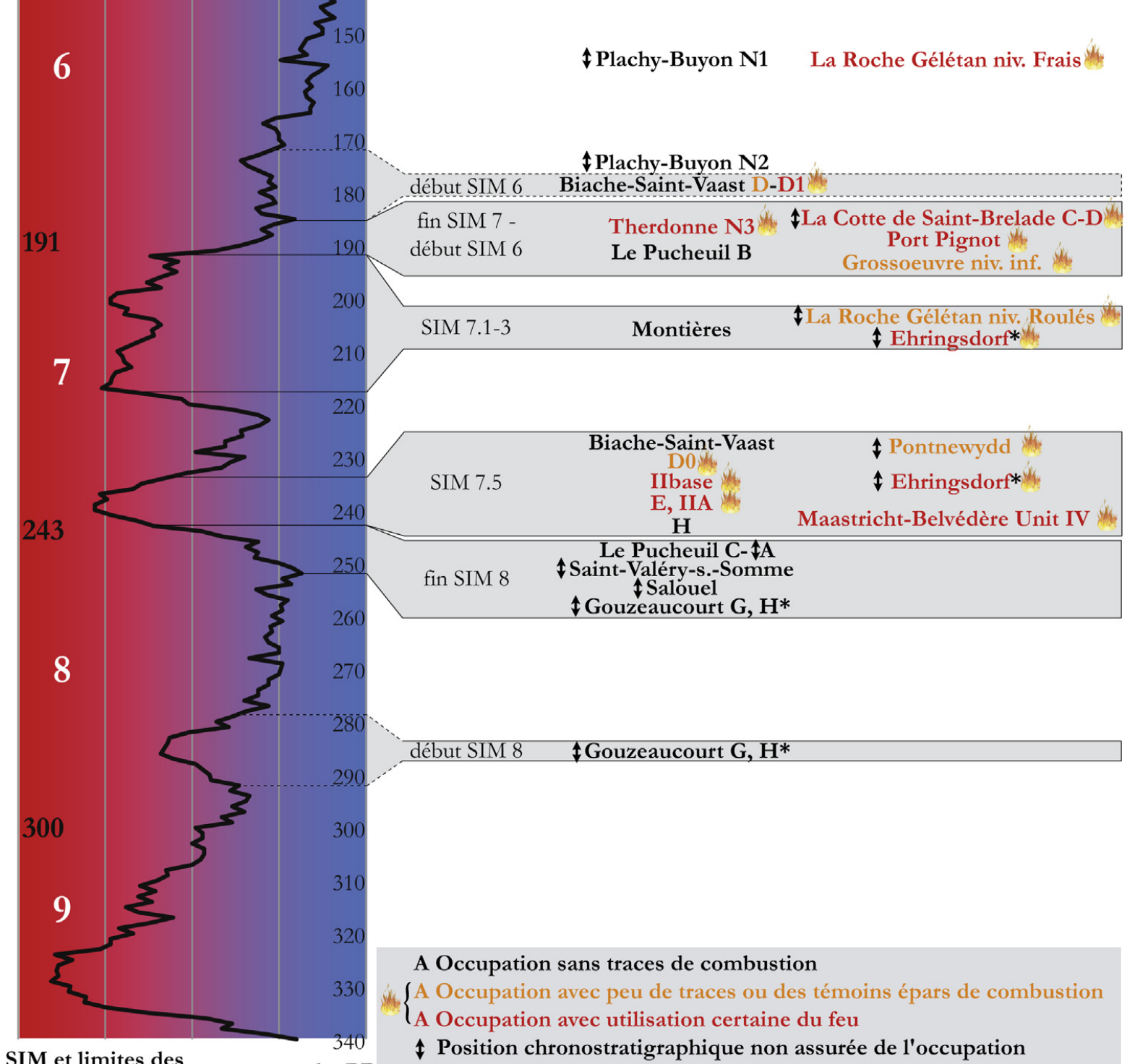

SIM et limites des

SIM en ka BP

ka BP

A Occupation sans traces de combustion

4. A Occupation avec peu de traces ou des témoins épars de combustion

A Occupation avec utilisation certaine du feu

$\uparrow$ Position chronostratigraphique non assurée de l'occupation

* Deux positions chronostratigraphiques possibles

Fig. 2. Principaux gisements saaliens (SIM 8 à 6 ; 300 à 130 ka BP) du Nord de la France et gisements saaliens du NordOuest de l'Europe possédant des traces de feu corrélés à la courbe isotopique marine LR04 (d'après Lisiecki et Raymo, 2005). Calage chronostratigraphique des occupations et informations sur les trace de feu d'après (Aldhouse-Green, 1995 ; Ameloot-Van der Heijden et al., 1996 ; Antoine, 1989 ; Behm-Blancke, 1960 ; Blondiau et al., 2009 ; Callow et Cornford, 1986 ; p. 193-195 ; Cliquet et Lautridou, 2009 ; Cliquet et al., 2003 ; Delagnes et Ropars, 1996 ; Green, 1983 ; Green et al., 1989 ; Heinzelin et Haesaerts, 1983 ; Hérisson, 2012 ; Hérisson et al., 2013 ; Hérisson et Locht, 2013 ; Locht et al., 1995, 2010 ; Mania et Mania, 2008 ; Roebroeks, 1988 ; Schäfer et Jäger, 1984 ; Stapert, 2007 ; Steiner, 1975 ; Tuffreau, 1987 ; Tuffreau et Bouchet, 1985 ; Tuffreau et Sommé, 1988a).

Main saalian sites (MIS 8 to 6; 300 to 130 ky BP) of Northern France and saalian sites of North-western Europe with fire traces correlated to isotopic marine curve LR04 (after Lisiecki et Raymo, 2005). Chronostratigraphical position of archaeological levels and datas about fire traces after (Aldhouse-Green, 1995; Ameloot-Van der Heijden et al., 1996; Antoine, 1989; Behm-Blancke, 1960; Blondiau et al., 2009; Callow et Cornford, 1986, p. 193-195; Cliquet et Lautridou, 
du Chantier Sud diffère des deux autres secteurs. Cinq niveaux archéologiques furent mis au jour : H, E, D0, D1 et D.

Le gisement de Therdonne (Fig. 1) fut découvert par J.-L. Locht durant le printemps 1998 à l'occasion d'une campagne de sondages archéologiques sur le tracé de la déviation de la RN 31 faisant la liaison entre Beauvais et Clermont (Oise), à la hauteur du village de Laversines, au pied de la butte tertiaire du Mont de Bourguillemont (Locht et al., 2010). Les sondages ont livré quatre niveaux archéologiques, nommés N1 à N4. Tenant compte des différents états de conservation des niveaux, l'objectif principal de l'opération préventive dirigé par J.-L. Locht d'août à novembre 1999 a été la fouille du niveau N3, très riche en artefacts lithiques et parfaitement conservés.

\subsection{Lithostratigraphie et position chronostratigrapique des niveaux saaliens de Biache- Saint-Vaast}

Huit niveaux archéologiques individualisés sur le gisement de Biache-Saint-Vaast sont étudiés ici : trois sont issus du chantier de 1976 (IIA, II $\alpha$, IIbase) et cinq du chantier Sud (H, E, D0, D1, D).

Dans le chantier de 1976, la séquence archéologique débute par le niveau IIA, contenu dans l'unité $2 \mathrm{~b}$ et situé « au-dessus de bancs de tuf durcis et de rubans violacés » (Tuffreau, 1988b, p. 123). Le niveau II $\alpha$ contenu dans l'unité $2 b$ « se situe quelques centimètres au-dessus du niveau IIA » (Tuffreau, 1988b). Le niveau II $\alpha$ est très certainement subcontemporain du niveau IIA sous-jacent. Le niveau II base est contenu dans le limon humifère brunâtre de l'unité 3a.

Dans le Chantier Sud, le plus ancien niveau archéologique H est contenu dans l'unité 2a. Le niveau E contenu dans l'unité $2 \mathrm{~b}$ occupe une position stratigraphique dans le chantier Sud comparable à celle du niveau IIA dans le chantier de 1976. L'hypothèse d'une subcontemporanéité des deux niveaux peut être avancée (Ameloot-Van der Heijden, 1991, p. 120). Le niveau D0 contenu dans l'unité $3 b$ est situé dans un « horizon grisâtre violacé, développé à la partie supérieure des formations fluviatiles » (Tuffreau, 1988b, p. 129). La séquence archéologique s'achève par la présence de l'unité 5 contenant le niveau $\mathrm{D}$ à sa base et D1 quelques centimètres à une vingtaine de centimètres dessus. Ces deux niveaux apparaissent eux aussi très proches dans le temps.

D'un point de vue paléoenvironnemental, «L[es] première[s] occupation[s] humaine[s] (niveaux I à D0) se situerai[en]t en fin de période interglaciaire ou interstadiaire très marquée, dans un milieu globalement dominé par la forêt mais où les espaces découverts de type prairie ou steppe demeurent toujours présents, sous un climat tempéré et nettement humide, plus continental que l'actuel ; cette première phase est suivie d'une période de péjoration climatique où l'Homme est absent du site ; la seconde [phase d'] loccupation[s] humaine[s] (niveaux D1 et D) succède à cette phase froide durant un épisode tempéré moins marqué que le précédent, où la forêt est beaucoup moins développée » (Auguste, 1995a, p. 489). La séquence saalienne de Biache-Saint-Vaast s'achève par une dégradation climatique aboutissant à la mise en place de conditions périglaciaires (Tuffreau et Sommé, 1988b, p. 118). La partie supérieure de la stratigraphie a notamment été complétée par les observations des profils de la paroi Est du Chantier Sud ainsi que dans les talus des voies ferrées de l'usine et de l'ancienne briqueterie

2009; Cliquet et al., 2003; Delagnes et Ropars, 1996; Green, 1983; Green et al., 1989; Heinzelin et Haesaerts, 1983; Hérisson, 2012; Hérisson et al., 2013; Hérisson et Locht, 2013; Locht et al., 1995, 2010; Mania et Mania, 2008; Roebroeks, 1988; Schäfer et Jäger, 1984; Stapert, 2007; Steiner, 1975; Tuffreau, 1987; Tuffreau et Bouchet, 1985; Tuffreau et Sommé, 1988a). 
proche montrant le développement du sol Eémien et de loess attribuables au Weichsélien (Sommé, 1988, p. 43-45).

La première phase d'occupations (niveaux H, IIA, II $\alpha$, IIbase, E, D0) serait donc contemporaine du début du stade isotopique 7 (SIM 7.5). Le hiatus d'occupation humaine marqué par la présence de l'unité stratigraphique 4 serait corrélé au refroidissement médian du stade isotopique 7 (SIM 7.4). La deuxième phase d'occupations (niveaux D1, D) daterait du début du stade isotopique 6 .

\subsection{Lithostratigraphie et position chronostratigrapique du niveau N3 de Therdonne}

À Therdonne, les formations quaternaires surmontent directement sur le substrat tertiaire (sable thanétien [SV]), sur lequel repose un niveau archéologique démantelé (N4). Elles débutent, à la base, par une unité de sable éolien gris-noir à brun, avec de nombreux petits nodules organo-ferriques (SGH). Il s'agit du remaniement par voie éolienne (déflation) du substrat sableux local, ensuite affecté par une pédogenèse humifère. Par sa structure, ce sol peut être rapproché des sols isohumiques steppiques du Début Glaciaire weichselien, contemporains de l'extrême fin du stade 5 (Antoine, 1989). Il contient le niveau principal N3, en position primaire (Locht et al., 2010). La position stratigraphique de ce sol, situé sous le sol interglaciaire eémien (LSABR), ainsi que les datations TL sur silex chauffés qui ont donné un âge de $178 \pm 11$ ka BP permettent de le placer, ainsi que le niveau archéologique N3 à la fin du stade isotopique 7.1 ou au tout début du stade 6. La nature de ce sol isohumique de type steppique permet de le rapprocher de ceux contemporains du Début Glaciaire weichselien. L'occupation principale de Therdonne a ainsi pris place en contexte de début de période froide, dans un environnement ouvert, sous des conditions plus continentales qu'actuellement. Ce paléosol est scellé par deux unités distinctes de sables ruisselés (SLBL et SAR). La première contient des artefacts lithiques en position remaniée. Au-dessus se trouve un limon sablo-argileux brun-rouge (LSABR), qui correspond à un horizon de type Bt de sol brun lessivé tronqué (interglaciaire eémien). Cette unité est entaillée par de grandes fentes à coin de glace, dont le remplissage est en partie constitué de limons humifères brun-grisâtre (RFG), provenant du démantèlement d'un sol gris-forestier (LSABH) et d'un sol isohumique de type steppique (LSAH). Ces deux horizons sont, latéralement, mal conservés en dehors du remplissage des fentes de gel. Les limons LSABH contiennent des artefacts lithiques en position remaniée (N1). La partie supérieure de l'unité LSAH a été tronquée par la mise en place d'un cailloutis de silex et de grès résultant de la phase d'érosion qui marque le début de l'unité supérieure (SGBL). Celle-ci est constituée de sables limoneux lités à niveaux argileux gris. Ensuite se trouve un limon sablo-argileux brun-grisâtre faiblement humifère (LSABG) d'origine éolienne à forte charge locale (déflation). Il est surmonté d'un limon sableux brun orangé, qui correspond à un sol brun arctique (LSABO). Audessus se trouve un limon grisâtre isohumique interprété comme un sol de prairie arctique (LBGH). La séquence se termine par des sables limoneux lités (SLBJ) surmontés d'un horizon limoneux brun argileux (LABR [Bt tardiglaciaire/Holocène]).

\section{L'utilisation du feu sur le gisement de Biache-Saint-Vaast}

\subsection{De rares mentions de l'utilisation du feu à Biache-Saint-Vaast}

La question de l'utilisation du feu sur le gisement de Biache-Saint-Vaast n'a jamais été clairement posée. Rares sont les mentions au feu dans les études antérieures. Pourtant, 
A. Tuffreau affirmait dès 1978 pour le niveau IIA que « l'utilisation du feu est attestée par la présence de nombreux fragments charbonneux, probablement dispersés dans le secteur le plus riche par des inondations, de silex, d'os et de grès brûlés. Un amas charbonneux ayant servi de foyer a été dégagé dans les m2 21 A, B, F, G » (Tuffreau, 1978, p. 49). P. Auguste fit également mention d'os brulés, entre autre, dans sa thèse sans approfondir la question du feu (Auguste, 1995a,b). Si les évocations publiées sont rares, les archives et photographies conservées sont aussi peu loquaces. Seuls un plan de détail du niveau IIA et un plan du niveau D1 trahissent la présence de charbons de bois dans ces niveaux (Marcy et Tuffreau, 1988, p. 264 ; Tuffreau, 1988a, p. 102). Côté archives, seule une lettre d'A. Tuffreau datée du 25 avril 1977 confirme la présence d' " assez nombreux charbons de bois » trouvés dans le niveau IIA. Nul autre document n'apporte clairement d'évidences, à l'instar de la zone précédemment décrite dont nous n'avons malheureusement retrouvé aucune photographie à ce jour. Cette absence de preuves formellement rapportées se traduit, en dehors du cercle des personnes ayant travaillé sur le gisement, par un fort doute quant à l'usage du feu dans les niveaux de Biache-Saint-Vaast (Roebroeks et Villa, 2011, cf. table de données en annexe de l'article). Ce constat montre l'intérêt d'une remise à plat des données disponibles concernant le feu à Biache-Saint-Vaast et de traquer tous les indices exploitables afin de livrer une image plus juste de son utilisation pour chaque niveau.

\subsection{Des indices exploitables sur la présence de feu}

L'inspection minutieuse des archives n'a malheureusement pas été fructueuse, à l'image de la recherche des prélèvements de l'époque de la fouille qui est restée vaine. Sans support visuel ni échantillon, il ne reste plus qu'à se tourner vers les derniers restes encore accessibles et pouvant fournir de l'information : les vestiges fauniques et lithiques. Au final, les ossements et les silex brûlés sont les derniers et uniques témoins pouvant attester de l'utilisation du feu dans les niveaux de Biache-Saint-Vaast. Face à ces indices ténus, il est raisonnable de restreindre le champ d'investigation à trois questions pour chaque niveau. D'après les indices en présence, les Néandertaliens ont-ils eu recours au feu ? Concernant les os brûlés, sont-ils représentatifs de l'assemblage des niveaux en terme d'espèce et certaines parties anatomiques ont-elles subi préférentiellement le feu ? Existe-il des zones de concentration de matériel brûlé pouvant suggérer l'emplacement d'un foyer?

L'analyse suivante par niveau présente les vestiges témoignant d'un usage du feu afin de répondre à ces questions. Les analyses de la faune brûlée ont été menées à partir des données recueillies par P. Auguste lors de sa thèse et son HDR encore inexploitées sous cet axe (Auguste, 1995a, 2012).

\subsection{Niveau $H$}

D'après la bibliographie et les archives, le niveau H n'a livré aucun charbon de bois et aucune zone de combustion n'a été découverte. Aucun reste faunique ne possède de trace de combustion. Aucun indice d'exposition au feu n'a été détecté dans l'assemblage lithique. Tous ces éléments plaident en faveur d'une absence totale d'utilisation du feu pour le niveau $\mathrm{H}$.

\subsection{Niveau IIA}

Comme évoqué précédemment, la question de la présence de feu dans le niveau IIA a été rapidement tranchée. «L'utilisation du feu est attestée par la présence de nombreux fragments 
charbonneux, probablement dispersés dans le secteur le plus riche par des inondations, de silex, d'os et de grès brûlés. Un amas charbonneux ayant servi de foyer a été dégagé dans les m2 $21 \mathrm{~A}$, $\mathrm{B}, \mathrm{F}, \mathrm{G}$ » (Tuffreau, 1978, p. 49). Malheureusement, les prélèvements de charbon et les grès brûlés mentionnés n'ont pas été retrouvés. Ces témoins riches en information n'ont donc pas pu être étudiés et intégrés dans notre analyse. La localisation d'un « secteur comprenant de nombreux charbons de bois » dans les grands carrés 15 et 21 a été repérée sur un schéma de détail d'une zone du niveau IIA (Fig. 3) (Tuffreau, 1988a, p. 102). Mis à part ces deux éléments publiés, les vestiges fauniques et lithiques livrent de précieux renseignements.

Plus de $1 \%$ des restes fauniques retrouvés ont été brûlés, soit 2040 éléments. La très grande majorité $(91 \%)$ de ces derniers n'a pu être déterminée en termes d'espèce (Tableau 1$)$. Les 190 restes brûlés déterminés appartiennent à 6 espèces sur les 20 reconnues dans le niveau IIA. Ces 6 espèces sont inégalement représentées puisque deux espèces dominent largement l'ensemble : Ursus sp. (41\% des os brûlés déterminés) et Bos primigenius (38\% des os brûlés déterminés). Trois autres espèces présentent quelques restes brûlés : Ursus arctos ( $9 \%$ des brûlés déterminés), Megaloceros giganteus (8\% des os brûlés déterminés) et Cervus elaphus (39\% des

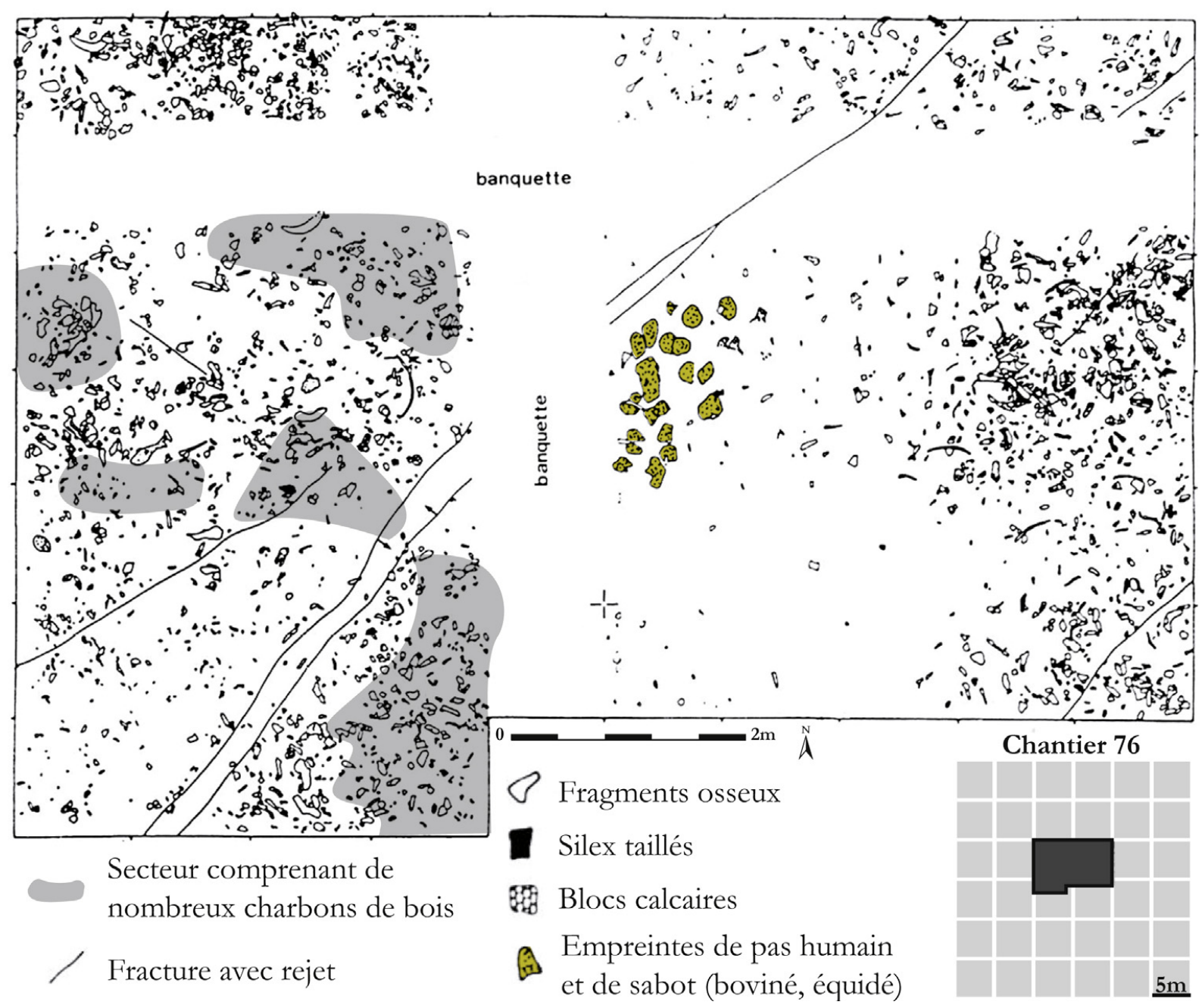

Fig. 3. Niveau IIA de Biache-Saint-Vaast. Secteur comprenant de nombreux charbons de bois dans les grands carrés 15 et 21 (Tuffreau et Sommé, 1988a, p. 102).

Level IIA of Biache-Saint-Vaast. Sector with numerous wood charcoal in huge scare 15 and 21 (Tuffreau et Sommé, 1988 , p. 102). 
Tableau 1

Niveau IIA de Biache-Saint-Vaast. Décompte et pourcentage des restes fauniques brûlés et non brûlés par espèce.

Level IIA of Biache-Saint-Vaast. Counting and percentage of burnt and non-burnt faunal remains by species.

\begin{tabular}{|c|c|c|c|c|c|c|c|c|}
\hline Espèces & NR & $\% \mathrm{NR}$ & NMI & $\% \mathrm{NMI}$ & NR brûlés & $\begin{array}{l}\% \text { du total du } \\
\text { NR brûlés }\end{array}$ & $\begin{array}{l}\text { \% du NR brûlés } \\
\text { indét. exclus }\end{array}$ & $\begin{array}{l}\text { \% du NR brûlés } \\
\text { de l'espèce }\end{array}$ \\
\hline Bos primigenius & 9188 & $47,3 \%$ & 158 & $34,1 \%$ & 73 & $3,58 \%$ & $38,4 \%$ & $0,79 \%$ \\
\hline Ursus arctos & 2158 & $11,1 \%$ & 75 & $16,2 \%$ & 18 & $0,88 \%$ & $9,5 \%$ & $0,83 \%$ \\
\hline Stephanorhinus hemitoechus & 1019 & $5,2 \%$ & 62 & $13,4 \%$ & 1 & $0,05 \%$ & $0,5 \%$ & $0,10 \%$ \\
\hline Ursus sp. & 4450 & $22,9 \%$ & 55 & $11,9 \%$ & 77 & $3,77 \%$ & $40,5 \%$ & $1,73 \%$ \\
\hline Stephanorhinus sp. & 1801 & $9,3 \%$ & 33 & $7,1 \%$ & 1 & $0,05 \%$ & $0,5 \%$ & $0,06 \%$ \\
\hline Ursus deningeri & 204 & $1,1 \%$ & 17 & $3,7 \%$ & & & & \\
\hline Stephanorhinus kirchbergensis & 109 & $0,6 \%$ & 15 & $3,2 \%$ & & & & \\
\hline Cervus elaphus & 139 & $0,7 \%$ & 13 & $2,8 \%$ & 5 & $0,25 \%$ & $2,6 \%$ & $3,60 \%$ \\
\hline Sus scrofa & 88 & $0,5 \%$ & 9 & $1,9 \%$ & 1 & $0,05 \%$ & $0,5 \%$ & $1,14 \%$ \\
\hline Megaloceros giganteus & 92 & $0,5 \%$ & 8 & $1,7 \%$ & 14 & $0,69 \%$ & $7,4 \%$ & $15,22 \%$ \\
\hline Equus cf achenheimensis & 74 & $0,4 \%$ & 5 & $1,1 \%$ & & & & \\
\hline Capreolus capreolus & 36 & $0,2 \%$ & 4 & $0,9 \%$ & & & & \\
\hline Aonyx antiqua & 19 & $0,1 \%$ & 2 & $0,4 \%$ & & & & \\
\hline Palaeoloxodon antiquus & 13 & $0,1 \%$ & 1 & $0,2 \%$ & & & & \\
\hline Equus hydruntinus & 13 & $0,1 \%$ & 1 & $0,2 \%$ & & & & \\
\hline Felis silvestris & 8 & $0,0 \%$ & 1 & $0,2 \%$ & & & & \\
\hline Panthera spelaea & 3 & $0,0 \%$ & 1 & $0,2 \%$ & & & & \\
\hline Canis lupus & 2 & $0,0 \%$ & 1 & $0,2 \%$ & & & & \\
\hline Martes cf martes & 2 & $0,0 \%$ & 1 & $0,2 \%$ & & & & \\
\hline Vulpes vulpes & 2 & $0,0 \%$ & 1 & $0,2 \%$ & & & & \\
\hline Total des déterminés & 19420 & $100 \%$ & 463 & $100 \%$ & 190 & $9,31 \%$ & $100 \%$ & - \\
\hline Indéterminés & 170340 & - & - & - & 1850 & $90,69 \%$ & & $1,09 \%$ \\
\hline Total & 189760 & - & - & - & 2040 & $100 \%$ & - & - \\
\hline
\end{tabular}


os brûlés déterminés). Les trois dernières espèces ne possèdent qu'un unique reste brûlé synonyme de présence anecdotique ou accidentelle : Stephanorhinus hemitoechus, Stephanorhinus sp. et Sus scrofa. La proportion de restes brûlés par espèce (Tableau 1) montre clairement que deux espèces ont fortement été affectées par le feu : Megaloceros giganteus (16\% des restes de l'espèce) et Cervus elaphus (3\% des restes de l'espèce). Ce phénomène est d'autant plus intéressant s'il est corrélé aux types d'ossements brûlés (Tableau 2). En effet, une véritable sélection est opérée dans les restes de Megaloceros giganteus et Cervus elaphus puisque seuls les bois des cervidés ont subi l'action du feu. Aucune autre partie anatomique présente de cervidé n'a subi d'altération thermique. Du côté d'Ursus sp. et de Bos primigenius, les os de la partie crâniale et les côtes dominent les autres parties anatomiques brûlées (Tableau 2).

Sur les 47150 pièces lithiques, 1373 artefacts ont subi des dégradations thermiques liées au feu, soit tout de même plus de 2,9\% de l'industrie (Tableau 3). Les catégories brûlées les plus présentes sont les éclats inférieurs à $3 \mathrm{~cm}$ (48\% des artefacts brûlés), les esquilles (35\% des artefacts brûlés) et les éclats non corticaux ( $8 \%$ des artefacts brûlés). Les éclats inférieurs à $3 \mathrm{~cm}$ est la seule catégorie surreprésentée dans les brûlés proportionnellement aux éléments non brûlés.

La répartition des vestiges brûlés lithiques et fauniques à Biache-Saint-Vaast est le reflet de la distribution générale des pièces non brûlées (Tableau 3). Que ce soit pour la faune ou le lithique, la distribution des mètres carrés ayant un pourcentage élevé d'éléments brûlés est directement corrélé à la répartition des zones à faible densité. Autrement dit, le pourcentage d'éléments brûlés est élevé dans ces carrés car la présence de peu d'éléments favorise un taux élevé. La distribution des éléments brûlés ne reflète donc pas celle de foyers sur la surface fouillée. Cet effet de flou peut s'expliquer par le phénomène de palimpseste du niveau précédemment évoqué. À cet effet palimpseste s'ajoute celui de l'existence de crues de la Scarpe qui ont tendance à redistribuer les éléments les plus légers altérés par la combustion, en particulier les os et les charbons. L'existence de foyers dans le niveau IIA est indéniable vu la quantité d'ossements et silex affectés par le feu ainsi que les charbons retrouvés. Leur localisation n'est cependant plus cernable à cause du remaniement de leurs résidus, dispersés par le lessivage des débordements successifs.

Tous les indices recueillis sont en accord avec les données issues de la fouille et convergent vers une utilisation contrôlée du feu par les Néandertaliens du niveau IIA. Aucun doute ne peut être émis sur l'origine anthropique du feu. Les études archéozoologiques montrent une nette différenciation de traitement entre les espèces. Seules cinq espèces ciblées semblent avoir été sélectionnées pour «passer au feu ». Il est intéressant de noter qu'une espèce comme le rhinocéros ne présente aucun reste altéré par le feu alors que de nombreux restes ont été retrouvés dans le niveau ( 2 fragments brûlés sur 2752 pièces ; phénomène anecdotique voir accidentel). L'hypothèse d'une cuisson alimentaire est envisagée pour les restes d'Ursus (sp. et arctos) et de Bos primigenius en corrélation avec les parties anatomiques considérées et les traces de découpes que les ossements portent : $28 \%$ des os brûlés avec des traces de découpe pour Ursus sp., $18 \%$ pour Bos primigenius et $53 \%$ pour Ursus arctos. Pour les cervidés, Megaloceros giganteus et Cervus elaphus, l'utilisation des bois comme combustible apparait une hypothèse peu probable vu la faible qualité comburante de ces parties anatomiques. Peut-être faudrait-il plutôt avancer la piste d'activités réalisées à proximité de foyers ou directement en interaction avec ces derniers, activités durant lesquelles les bois de cervidés seraient impliqués.

\subsection{Niveau IIbase}

Pour le niveau IIbase, aucune mention de charbon de bois ou de zone de combustion n'a été référencée. 
Tableau 2

Niveau IIA de Biache-Saint-Vaast. Inventaire des restes fauniques brûlés par espèce.

Level IIA of Biache-Saint-Vaast. Inventory of burnt faunal remains by species.

\begin{tabular}{|c|c|c|}
\hline Espèces Os & NR brûlés & $\%$ \\
\hline Ursus sp. & 77 & \\
\hline Côtes & 31 & 40,3 \\
\hline Crânes & 17 & 22,1 \\
\hline Phalanges & 7 & 9,1 \\
\hline Vertèbres & 4 & 5,2 \\
\hline Mandibules & 3 & 3,9 \\
\hline Humérus & 2 & 2,6 \\
\hline Métapodes & 2 & 2,6 \\
\hline Scapulas & 2 & 2,6 \\
\hline Sésamoïdes & 2 & 2,6 \\
\hline Tibias & 2 & 2,6 \\
\hline Calcaneus & 1 & 1,3 \\
\hline Fibula & 1 & 1,3 \\
\hline Trapèze & 1 & 1,3 \\
\hline Ulna & 1 & 1,3 \\
\hline Métacarpe & 1 & 1,3 \\
\hline Bos primigenius & 72 & \\
\hline Crânes & 27 & 37,5 \\
\hline Côtes & 25 & 34,7 \\
\hline Mandibules & 4 & 5,6 \\
\hline Vertèbres & 4 & 5,6 \\
\hline Tibias & 3 & 4,2 \\
\hline Phalanges & 2 & 2,8 \\
\hline Radio-ulnas & 2 & 2,8 \\
\hline Cubo-naviculaire & 1 & 1,4 \\
\hline Fémur & 1 & 1,4 \\
\hline Métatarse & 1 & 1,4 \\
\hline Radius & 1 & 1,4 \\
\hline Ulna & 1 & 1,4 \\
\hline Ursus arctos & 17 & \\
\hline Phalanges & 9 & 52,9 \\
\hline Crâne & 1 & 5,9 \\
\hline Mandibule & 1 & 5,9 \\
\hline Métacarpe & 1 & 5,9 \\
\hline Patella & 1 & 5,9 \\
\hline Radius & 1 & 5,9 \\
\hline Scapula & 1 & 5,9 \\
\hline Trapézoïde & 1 & 5,9 \\
\hline Ulna & 1 & 5,9 \\
\hline Megaloceros giganteus & 14 & \\
\hline Bois & 14 & 100 \\
\hline Cervus elaphus & 5 & \\
\hline Bois & 5 & 100 \\
\hline Stephanorhinus hemitoecus & 1 & \\
\hline Fémur & 1 & 100 \\
\hline Stephanorhinus sp. & 1 & \\
\hline Vertèbre & 1 & 100 \\
\hline Sus scrofa & 1 & \\
\hline Mandibule & 1 & 100 \\
\hline
\end{tabular}


Tableau 3

Niveau IIA de Biache-Saint-Vaast. Décompte et pourcentage des différentes catégories technologiques d'artefacts lithiques brûlés et non brûlés.

Level IIA of Biache-Saint-Vaast. Counting and percentage of different technological categories of burnt and non-burnt lithic artifacts.

\begin{tabular}{lrlcc}
\hline Catégories & $\begin{array}{l}\text { NR } \\
\text { brûlés }\end{array}$ & $\begin{array}{l}\% \text { du total } \\
\text { du NR brûlés }\end{array}$ & $\begin{array}{l}\text { NR total } \\
\text { (brûlés et non brûlés) }\end{array}$ & $\begin{array}{l}\% \text { du NR brûlés } \\
\text { de chaque catégorie }\end{array}$ \\
\hline Éclats Levallois & 32 & $2,3 \%$ & 2281 & $1,4 \%$ \\
Pointes Levallois & 0 & - & 2 & - \\
Éclats Kombewa & 1 & $0,1 \%$ & 125 & $0,8 \%$ \\
Éclats non corticaux & 112 & $8,2 \%$ & 4546 & $2,5 \%$ \\
Éclats semi-corticaux & 48 & $3,5 \%$ & 3192 & $1,5 \%$ \\
Éclats corticaux & 34 & $2,5 \%$ & 1933 & $1,8 \%$ \\
Entame & 0 & - & 33 & - \\
Éclats caractéristiques & 1 & $0,1 \%$ & 120 & $0,8 \%$ \\
Nucléus & 1 & $0,1 \%$ & 381 & $0,3 \%$ \\
Cassons & 4 & $0,3 \%$ & 134 & $3,0 \%$ \\
Cassons gélivés & 1 & $0,1 \%$ & 1027 & $0,1 \%$ \\
Éclats $<3$ cm & 661 & $48,1 \%$ & 11320 & $5,8 \%$ \\
Esquilles & 478 & $34,8 \%$ & 22050 & $2,2 \%$ \\
Blocs testés & 0 & - & 3 & - \\
Percuteurs & 0 & - & 3 & - \\
Total & 1373 & $100 \%$ & 47150 & $2,9 \%$ \\
\hline
\end{tabular}

Un taux élevé de restes fauniques a été brûlé puisque 45 restes sur 628 ont subi une dégradation thermique, soit plus de $7 \%$ de l'assemblage (Tableau 4). La grande majorité (84\%) des vestiges brûlés n'a pas pu être déterminée en termes d'espèce. Les restes brûlés déterminés sont peu nombreux $(\mathrm{NR}=7)$ et appartiennent à 3 espèces sur 12 reconnues dans le niveau IIbase. La faiblesse numérique de l'assemblage brûlé identifié ne permet pas d'aller plus loin dans

Tableau 4

Niveau IIbase de Biache-Saint-Vaast. Décompte et pourcentage des restes fauniques brûlés et non brûlés par espèce. Level IIbase of Biache-Saint-Vaast. Counting and percentage of burnt and non-burnt faunal remains by species.

\begin{tabular}{|c|c|c|c|c|c|c|c|c|}
\hline Espèces & NR & $\% \mathrm{NR}$ & NMI & $\begin{array}{l}\% \\
\text { NMI }\end{array}$ & $\begin{array}{l}\text { NR } \\
\text { brûlés }\end{array}$ & $\begin{array}{l}\% \mathrm{du} \\
\text { total du } \\
\text { NR brûlés }\end{array}$ & $\begin{array}{l}\text { \% du NR } \\
\text { brûlés indét. } \\
\text { exclus }\end{array}$ & $\begin{array}{l}\text { \% du NR } \\
\text { brûlés de } \\
\text { l'espèce }\end{array}$ \\
\hline Bos primigenius & 268 & $53,5 \%$ & 10 & $25,6 \%$ & 5 & $11,1 \%$ & $71 \%$ & $1,87 \%$ \\
\hline Ursus sp. & 58 & $11,6 \%$ & 5 & $12,8 \%$ & 1 & $2,2 \%$ & $14 \%$ & $1,72 \%$ \\
\hline Cervus elaphus & 33 & $6,6 \%$ & 5 & $12,8 \%$ & & & & \\
\hline Stephanorhinus sp. & 37 & $7,4 \%$ & 4 & $10,3 \%$ & & & & \\
\hline Stephanorhinus hemitoechus & 21 & $4,2 \%$ & 4 & $10,3 \%$ & & & & \\
\hline Equus cf achenheimensis & 26 & $5,2 \%$ & 3 & $7,7 \%$ & & & & \\
\hline Ursus arctos & 30 & $6,0 \%$ & 2 & $5,1 \%$ & & & & \\
\hline Ursus deningeri & 17 & $3,4 \%$ & 2 & $5,1 \%$ & 1 & $2,2 \%$ & $14 \%$ & $5,88 \%$ \\
\hline Equus hydruntinus & 7 & $1,4 \%$ & 2 & $5,1 \%$ & & & & \\
\hline Stephanorhinus kirchbergensis & 4 & $0,8 \%$ & 2 & $5,1 \%$ & & & & \\
\hline Capreolus capreolus & 12 & $2,4 \%$ & 1 & $2,6 \%$ & & & & \\
\hline Megaloceros giganteus & 1 & $0,2 \%$ & 1 & $2,6 \%$ & & & & \\
\hline Total des déterminés & 501 & $100 \%$ & 39 & $100 \%$ & 7 & $15,6 \%$ & $100 \%$ & $1,21 \%$ \\
\hline Indéterminés & 3209 & & & & 38 & $84,4 \%$ & & $1,18 \%$ \\
\hline Total & 3710 & & & & 45 & $100 \%$ & & \\
\hline
\end{tabular}


Tableau 5

Niveau IIbase de Biache-Saint-Vaast. Inventaire des restes fauniques brûlés par espèce.

Level IIA of Biache-Saint-Vaast. Inventory of burnt faunal remains by species.

\begin{tabular}{lll}
\hline Espèces os & NR & $\%$ \\
\hline Bos primigenius & 5 & \\
Tibias & 2 & 40 \\
Fémur & 1 & 20 \\
Mandibule & 1 & 20 \\
Radius & 1 & 20 \\
Ursus deningeri & 1 & 100 \\
Calcaneus & 1 & 100 \\
Ursus sp. & 1 & \\
Crâne & 1 & \\
\hline
\end{tabular}

l'interprétation (Tableau 5). Il est tout de même intéressant de souligner qu'à l'image du niveau IIA, les restes de Bos primigenius et des ours (Ursus deningeri et sp.) ont été brûlés tandis que ceux des rhinocéros n'ont absolument pas subi le feu.

Sur les 4826 pièces lithiques, 79 artefacts ont subi des dégradations thermiques liées au feu, soit 1,6\% de l'industrie (Tableau 6). Les catégories brûlées les plus présentes sont les éclats inférieurs à $3 \mathrm{~cm}$ ( $52 \%$ des artefacts brûlés), les esquilles (35\% des artefacts brûlés) et les éclats non corticaux ( $8 \%$ des artefacts brûlés). Les deux catégories les plus présentes sont aussi surreprésentées dans les pièces brûlées proportionnellement aux éléments non brûlés.

La répartition des vestiges brûlés lithiques et fauniques à Biache-Saint-Vaast est le reflet à première vue de la distribution générale des pièces non brûlées (Fig. 4). Lorsque l'attention se porte en détail sur les différents plans de répartition, quatre zones de l'ordre d'un à trois mètres carrés constitués d'éléments fauniques et lithiques brûlés ressortent (Fig. 4). Deux autres mètres

Tableau 6

Niveau IIbase de Biache-Saint-Vaast. Décompte et pourcentage des différentes catégories technologiques d'artefacts lithiques brûlés et non brûlés.Level IIbase of Biache-Saint-Vaast.

Counting and percentage of different technological categories of burnt and non-burnt lithic artifacts.

\begin{tabular}{lllcl}
\hline Catégories & $\begin{array}{l}\text { NR } \\
\text { brûlés }\end{array}$ & $\begin{array}{l}\% \text { du total du } \\
\text { NR brûlés }\end{array}$ & $\begin{array}{l}\text { NR total } \\
\text { (brûlés et non brûlés) }\end{array}$ & $\begin{array}{l}\% \text { du NR brûlés } \\
\text { de chaque catégorie }\end{array}$ \\
\hline Éclats Levallois & 0 & - & 156 & - \\
Pointes Levallois & 0 & - & 0 & - \\
Éclats Kombewa & 0 & - & 7 & - \\
Éclats non corticaux & 6 & $7,6 \%$ & 878 & $0,7 \%$ \\
Éclats semi-corticaux & 2 & $2,5 \%$ & 434 & $0,5 \%$ \\
Éclats corticaux & 1 & $1,3 \%$ & 260 & $0,4 \%$ \\
Entame & 1 & $1,3 \%$ & 5 & $20,0 \%$ \\
Éclats caractéristiques & 0 & - & 15 & - \\
Nucléus & 0 & - & 75 & - \\
Cassons & 0 & - & 58 & - \\
Cassons gélivés & 0 & - & 95 & - \\
Éclats $<3$ cm & 41 & $51,9 \%$ & 1784 & $2,3 \%$ \\
Esquilles & 28 & $35,4 \%$ & 1054 & $2,7 \%$ \\
Blocs testés & 0 & - & 3 & - \\
Percuteurs & 0 & - & 2 & - \\
Total & 79 & $100 \%$ & 4826 & $1,6 \%$ \\
\hline
\end{tabular}


A
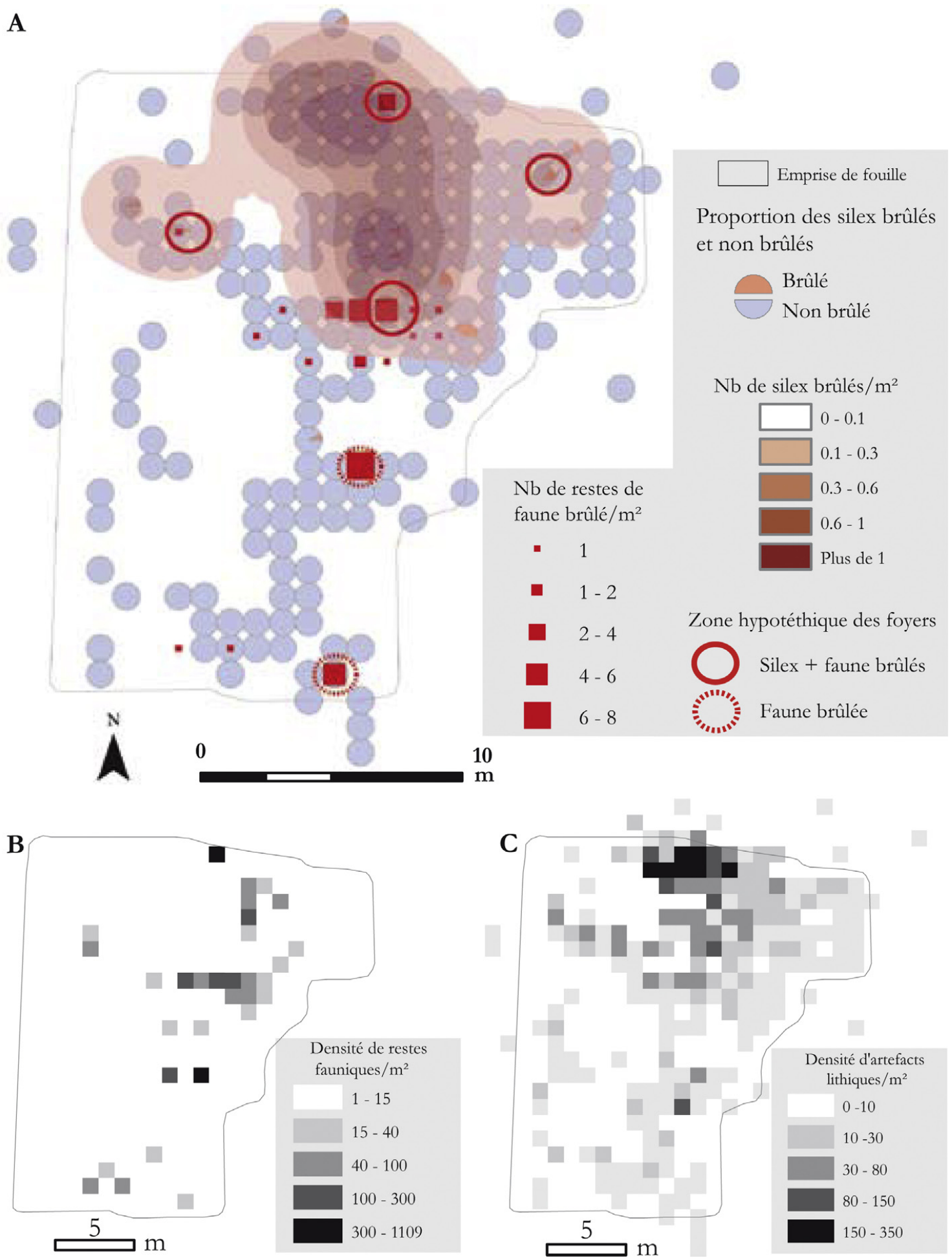

Fig. 4. Niveau IIbase de Biache-Saint-Vaast. A. Superposition de différents plans relatifs à l'impact du feu sur les vestiges fauniques et lithiques - plan de répartition par pourcentage des silex brûlés et non brûlés par mètre carré, plan d'isodensité des silex brûlés par mètre carré, plan de répartition ponctuelle des restes fauniques brûlés et zones hypothétiques des foyers. B. Plan de densité par mètre carré de l'ensemble des restes fauniques. C. Plan de densité par mètre carré de l'ensemble des artefacts lithiques.

Level IIbase of Biache-Saint-Vaast. A. Superimposition of different maps concerning fire impact on faunal remains and lithic artifacts - distribution per percentage of burnt and non-burnt flint per square meter, isodensity map of burnt fint per square meter, pointwise distribution of burnt faunal remains and hypothetic zones of hearth. B. Density map of all faunal remains per square meter. C. Density map of all lithic artifacts per square meter. 
carrés présentent de nombreux restes fauniques brûlés et peuvent aussi être considérés comme des zones potentielles de localisation de structure de combustion (Fig. 4). En résumé, cette brève analyse montre une corrélation entre des zones de forte densité des restes lithiques et fauniques et des zones de localisation hypothétique de foyers en périphérie directe, attestées par des restes brûlés de faune et de silex taillés.

Les indices recueillis ne laissent aucun doute quant à l'utilisation du feu par les Néandertaliens du niveau IIbase. L'élément le plus remarquable est la proportion élevée de restes fauniques brûlés. L'autre point à souligner est le spectre très réduit des espèces dont des restes ont été dégradés par le feu en regard de la richesse de l'assemblage faunique.

\subsection{Niveau $E$}

Pour le niveau E, aucune mention de charbon de bois ou de zone de combustion n'a été trouvée. Côté vestiges, seuls 9 restes fauniques indéterminés ont été brûlés sur les 1215 indéterminés retrouvés, soit $0,7 \%$. Aucun reste déterminé ne possède de stigmate de chauffe. Pour l'assemblage lithique, 117 artefacts ont été brûlés, soit 1,6\% de l'ensemble (Tableau 7). Aucun éclat Levallois n'a subi d'altération thermique liée au feu. Les esquilles, les éclats non corticaux et les cassons sont les trois catégories les plus affectées par le feu et de manière proportionnellement plus importante que pour les autres catégories. À l'inverse, les éclats inférieurs à $3 \mathrm{~cm}$, les éclats corticaux et les cassons gélivés sont sous représentés dans les éléments brûlés.

En dehors des vestiges, aucun élément ne permet d'attester la présence de feu dans le niveau E. Cependant, le taux d'artefacts lithiques brûlés et les quelques restes fauniques brûlés témoignent

Tableau 7

Niveau E de Biache-Saint-Vaast. Décompte et pourcentage des différentes catégories technologiques d'artefacts lithiques brûlés et non brûlés.

Level E of Biache-Saint-Vaast. Counting and percentage of different technological categories of burnt and non-burnt lithic artifacts.

\begin{tabular}{lclcc}
\hline Catégories & NR brûlés & $\begin{array}{l}\% \text { du total } \\
\text { du NR brûlés }\end{array}$ & $\begin{array}{l}\text { NR total (brûlés } \\
\text { et non brûlés) }\end{array}$ & $\begin{array}{l}\% \text { du NR brûlés } \\
\text { de chaque catégorie }\end{array}$ \\
\hline Éclats Levallois & 0 & - & 232 & - \\
Pointes Levallois & 0 & - & 0 & - \\
Éclats Kombewa & 0 & - & 10 & $2,0 \%$ \\
Éclats non corticaux & 17 & $14,5 \%$ & 847 & $1,6 \%$ \\
Éclats semi-corticaux & 7 & $6,0 \%$ & 449 & $0,9 \%$ \\
Éclats corticaux & 3 & $2,6 \%$ & 339 & $1,9 \%$ \\
Entame & 1 & $0,9 \%$ & 53 & - \\
Éclats caractéristiques & 0 & - & 65 & $0,9 \%$ \\
Nucléus & 1 & $0,9 \%$ & 116 & $2,2 \%$ \\
Cassons & 5 & $4,3 \%$ & 231 & - \\
Cassons gélivés & 0 & - & 139 & $1,3 \%$ \\
Éclats $<3$ cm & 39 & $33,3 \%$ & 3081 & $2,3 \%$ \\
Esquilles & 44 & $37,6 \%$ & 1913 & - \\
Blocs testés & 0 & - & 3 & - \\
Percuteurs & 0 & - & 5 & $1,6 \%$ \\
Total & 117 & $100 \%$ & 7483 & \\
\hline
\end{tabular}


de manière incontestable de la présence de feu dans le niveau. Les modalités de son utilisation restent inconnues à cause du peu d'indices disponibles pour les reconstituer.

\subsection{Niveau $\mathrm{DO}$}

Concernant le niveau D0, nulle mention de charbon de bois ou de zone de combustion n'a été recensée. Un unique reste indéterminé a subi le feu, soit $0,19 \%$ de l'ensemble des vestiges fauniques du niveau. Du point de vue de l'industrie lithique, 11 artefacts ont été brûlés, soit 0,8\% de l'ensemble (Tableau 8).

Les témoins de la présence du feu dans le niveau D0 sont rares mais existants. Devant la faiblesse de la documentation, il est difficile de trancher sur le statut des éléments brûlés et les modalités de l'usage du feu dans ce niveau.

\subsection{Niveau D1}

Sur le niveau D1, la présence de charbons de bois est notifiée sur un plan de répartition des vestiges (Marcy et Tuffreau, 1988, p. 264). Aucun reste faunique ne possède de trace de combustion. Du côté de l'assemblage lithique, seules 25 pièces ont été brûlées, soit $0,8 \%$ de l'ensemble (Tableau 9). Les éclats inférieurs à $3 \mathrm{~cm}$ et les éclats corticaux sont surreprésentés dans les éléments brûlés.

Malgré la présence de charbon de bois, il paraît aventureux de définir l'origine des dégradations thermiques observées et de déterminer les modalités d'utilisation du feu devant le peu de documentation recueillie. L'hypothèse la plus probable reste une origine anthropique du feu ayant entraîné l'altération des pièces brûlées retrouvées.

\section{Tableau 8}

Niveau D0 de Biache-Saint-Vaast. Décompte et pourcentage des différentes catégories technologiques d'artefacts lithiques brûlés et non brûlés.

Level DO of Biache-Saint-Vaast. Counting and percentage of different technological categories of burnt and non-burnt lithic artifacts.

\begin{tabular}{lllcl}
\hline Catégories & NR brûlés & $\begin{array}{l}\% \text { du total du } \\
\text { NR brûlés }\end{array}$ & $\begin{array}{l}\text { NR total (brûlés } \\
\text { et non brûlés) }\end{array}$ & $\begin{array}{l}\% \text { du NR brûlés } \\
\text { de chaque catégorie }\end{array}$ \\
\hline Éclats Levallois & 0 & - & 44 & - \\
Pointes Levallois & 0 & - & 0 & - \\
Éclats Kombewa & 0 & - & 1 & - \\
Éclats non corticaux & 1 & $9,1 \%$ & 157 & $0,6 \%$ \\
Éclats semi-corticaux & 1 & $9,1 \%$ & 123 & $0,8 \%$ \\
Éclats corticaux & 0 & - & 86 & - \\
Entame & 0 & - & 3 & - \\
Éclats caractéristiques & 0 & - & 2 & - \\
Nucléus & 0 & - & 21 & $5,3 \%$ \\
Cassons & 1 & $9,1 \%$ & 19 & - \\
Cassons gélivés & 0 & - & 68 & - \\
Éclats $<3$ cm & 8 & $72,7 \%$ & 658 & - \\
Esquilles & 0 & - & 221 & - \\
Blocs testés & 0 & - & 3 & $0,8 \%$ \\
Percuteurs & 0 & - & 1 & \\
Total & 11 & $100 \%$ & 1407 & \\
\hline
\end{tabular}


Tableau 9

Niveau D1 de Biache-Saint-Vaast. Décompte et pourcentage des différentes catégories technologiques d'artefacts lithiques brûlés et non brûlés.

Level D1 of Biache-Saint-Vaast. Counting and percentage of different technological categories of burnt and non-burnt lithic artifacts.

\begin{tabular}{lllcl}
\hline Catégories & NR brûlés & $\begin{array}{l}\% \text { du total du } \\
\text { NR brûlés }\end{array}$ & $\begin{array}{l}\text { NR total (brûlés } \\
\text { et non brûlés) }\end{array}$ & $\begin{array}{l}\% \text { du NR brûlés } \\
\text { de chaque catégorie }\end{array}$ \\
\hline Éclats Levallois & 0 & - & 207 & - \\
Pointes Levallois & 0 & - & 0 & - \\
Éclats Kombewa & 0 & - & 3 & - \\
Éclats non corticaux & 1 & $4 \%$ & 453 & - \\
Éclats semi-corticaux & 0 & - & 322 & $1,7 \%$ \\
Éclats corticaux & 4 & $16 \%$ & 240 & - \\
Entame & 0 & - & 15 & - \\
Éclats caractéristiques & 0 & - & 35 & - \\
Nucléus & 0 & - & 94 & - \\
Cassons & 0 & - & 25 & - \\
Cassons gélivés & 0 & - & 313 & $1,4 \%$ \\
Éclats $<3$ cm & 19 & $76 \%$ & 1352 & $0,9 \%$ \\
Esquilles & 1 & $4 \%$ & 113 & - \\
Blocs testés & 0 & - & 5 & - \\
Percuteurs & 0 & - & 6 & $0,8 \%$ \\
Total & 25 & $100 \%$ & 3183 & \\
\hline
\end{tabular}

\subsection{Niveau $D$}

Pour le niveau D, aucune mention de charbon de bois ou de zone de combustion n'a été trouvée. Aucun reste faunique ne possède de trace de combustion. Pour l'industrie lithique, seuls 5 artefacts ont été brûlés, soit à peine $1 \%$ de l'ensemble (Tableau 10).

À l'image du niveau D0, il est difficile de trancher sur le statut des éléments brûlés et les modalités de l'usage du feu dans ce niveau eu égard à la faiblesse de la documentation.

\section{L'usage du feu sur le gisement de Therdonne}

\subsection{De multiples questionnements liés à la découverte de zones de combustion}

Si l'origine anthropique des zones de combustion observées fut rapidement confirmée lors de la phase de fouilles (Locht et al., 2000, p. 45), cette découverte ouvrit la voie à de multiples questionnements. C'est en effet un véritable foisonnement de questions qui se bouscula lorsque nous entreprîmes de nous attaquer à ce volet du gisement :

- quelle est la nature exacte des zones de combustion observées à la fouille ? Foyers en place, vidanges de foyers, résidus de foyers dégradés, produits taphonomiques ?

- combien de zones ou plus précisément de structures de combustion peuvent être identifiées ?

- quel était leur mode de fonctionnement ? Type d'aménagement ? Combustibles utilisés ?

- quelle fut la fonction des structures de combustion ? Type d'utilisation ? Place dans l'occupation?

- quelle fut la durée d'utilisation des structures de combustion? Utilisation unique ou multiple ?

Combustion courte ou longue? 


\subsection{Quelle méthode employer pour répondre à ces questions et reconstituer l'utilisation du} feu à Therdonne?

Afin de canaliser ce foisonnement d'ardentes questions et d'appréhender le potentiel d'investigation, la première étape fut de dresser un bilan bibliographique des études de foyer préhistorique, des méthodes employées et des résultats obtenus (principalement Cliquet, 1992 ; Evershed, 1993 ; Lucquin, 2007 ; March, 1995, 1996 ; March et al., 2006 ; Meignen et al., 2000 ; Olive et Taborin, 1989 ; Perlès, 1977 ; Théry-Parisot, 2001). Les méthodes les plus couramment utilisées dans les études étaient inapplicables dans le cas de Therdonne. L'approche anthracologique ne pouvait être menée à cause du trop faible nombre de charbons retrouvés, leurs dimensions trop réduites et la mauvaise préservation de leurs structures. Il en était de même pour la micromorphologie puisque nous ne possédions pas de prélèvements en bloc mais simplement du sédiment prélevé en vrac.

La seule méthode qui est apparue envisageable pour apporter des éléments de réponse concernant la fonction et le fonctionnement des zones de combustion était l'analyse chimique organique. C'est ainsi que fut opéré un rapprochement avec A. Lucquin et R. March du laboratoire de Rennes afin de connaître la faisabilité d'une telle approche dans le cadre de Therdonne. Nous avons bénéficié de leur expertise et de leur savoir-faire concernant l'étude des structures de combustion. Cela nous a permis de définir une méthodologie appropriée dans le but de récupérer un maximum d'informations des données à notre disposition.

Quatre axes de recherche ont été définis :

- une reprise et un traitement des observations de terrain afin de caractériser la nature et les caractéristiques des zones de combustion mises au jour ;

- une reprise et un traitement des observations issues du foyer expérimental pour notamment appréhender la signature engendrée par la combustion sur le sédiment du site ;

- une étude des blocs calcaires et des silex brûlés en vue d'obtenir des indices concernant la fonction et le fonctionnement des zones de combustion et des activités environnantes dans l'occupation ;

- une analyse chimique des résidus organiques contenus dans les prélèvements archéologiques, expérimentaux et du sol encaissant afin de caractériser la fonction et le fonctionnement des zones de combustion.

Enfin, l'ensemble des données acquises par ces recherches a été synthétisé et intégré à l'analyse spatiale dans le but de définir la localisation des structures de combustion et d'appréhender le rôle joué par ces dernières dans l'occupation.

\subsection{Observations de terrain concernant la nature des zones de combustion}

Lors de la fouille, cinq zones de sédiment noirci ont été observées (Fig. 5). Deux zones de sédiment noirci plus réduites ont été relevées au sud de la fouille, dans les carrés B/C 94 et ZZ/A 94. À ce sédiment noirci, s'ajoutent des zones plus localisées de concentration en charbons de bois et en éléments brûlés dont les carrés H100 (Fig. 6) ou E104 (Fig. 7) sont représentatifs. Une zone s'étendant sur les carrés F101 et F102 présentait une très forte concentration en sédiment noirci, en charbons de bois et en éléments brûlés (Fig. 8). Cette dernière est incontestablement le témoin de combustion le mieux préservé de l'occupation. 


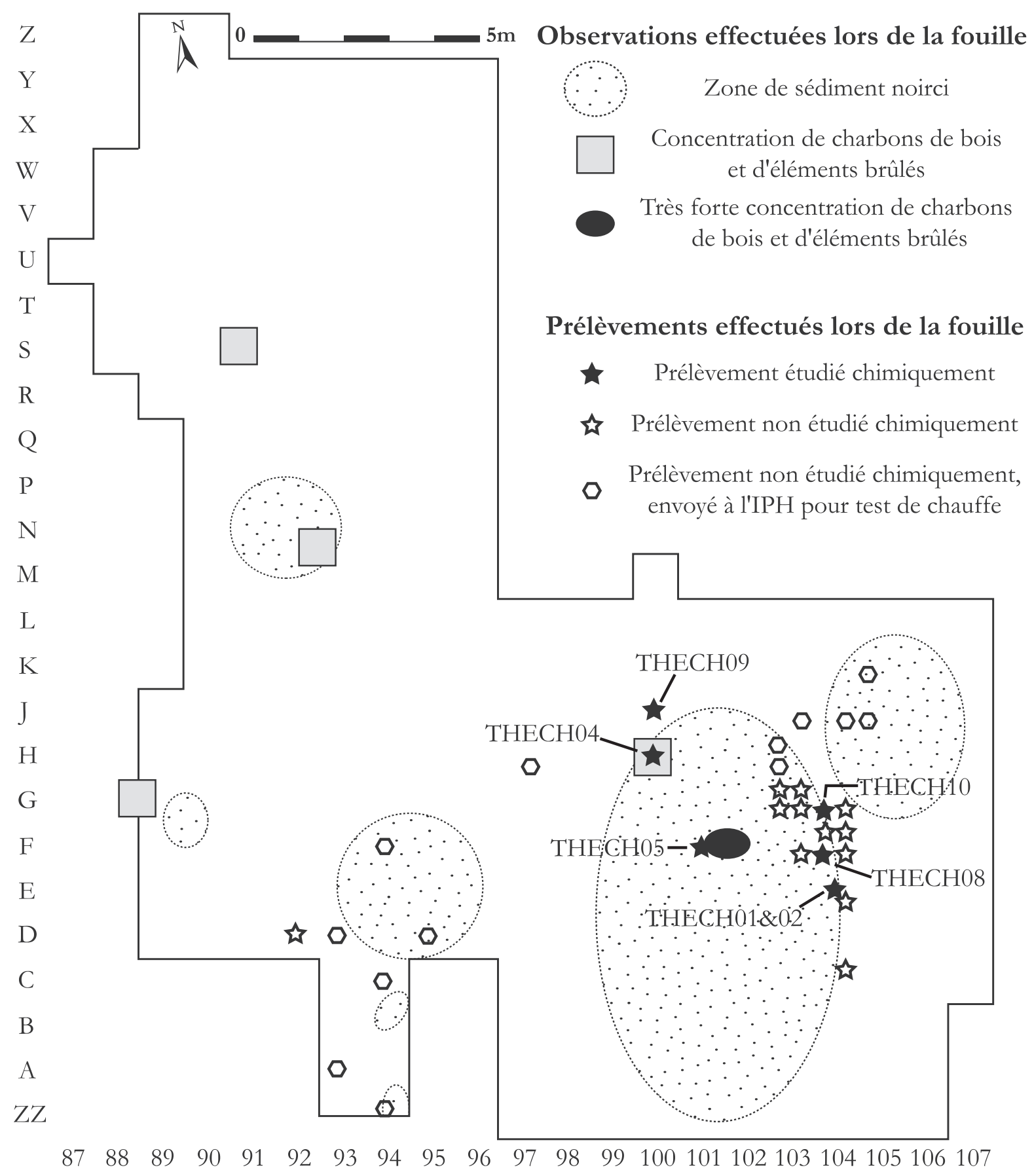

Fig. 5. Niveau N3 de Therdonne. Plan de localisation des observations et des prélèvements effectués concernant les vestiges de combustion.

Level N3 of Therdonne. Location map of observations and samples made concerning combustion remains.

Les concentrations de sédiment noirci, de charbons de bois et d'éléments brûlés présentaient des limites floues et n'avaient pas de forme identifiable (Fig. 5). Les charbons retrouvés étaient en très faible nombre et possédaient des dimensions très réduites ( $<1 \mathrm{~mm}$ dans la majorité des cas). Moins d'une dizaine de fragments d'os brûlés de petites dimensions ont été mis au jour, dont le mieux préservé se situait dans le carré J100 (Fig. 9). Aucun aménagement particulier n'a été détecté dans les zones noircies. Seuls des blocs calcaires de petites dimensions brûlés ont été retrouvés à proximité des zones de combustion. Dans tous les cas, si un aménagement simple 


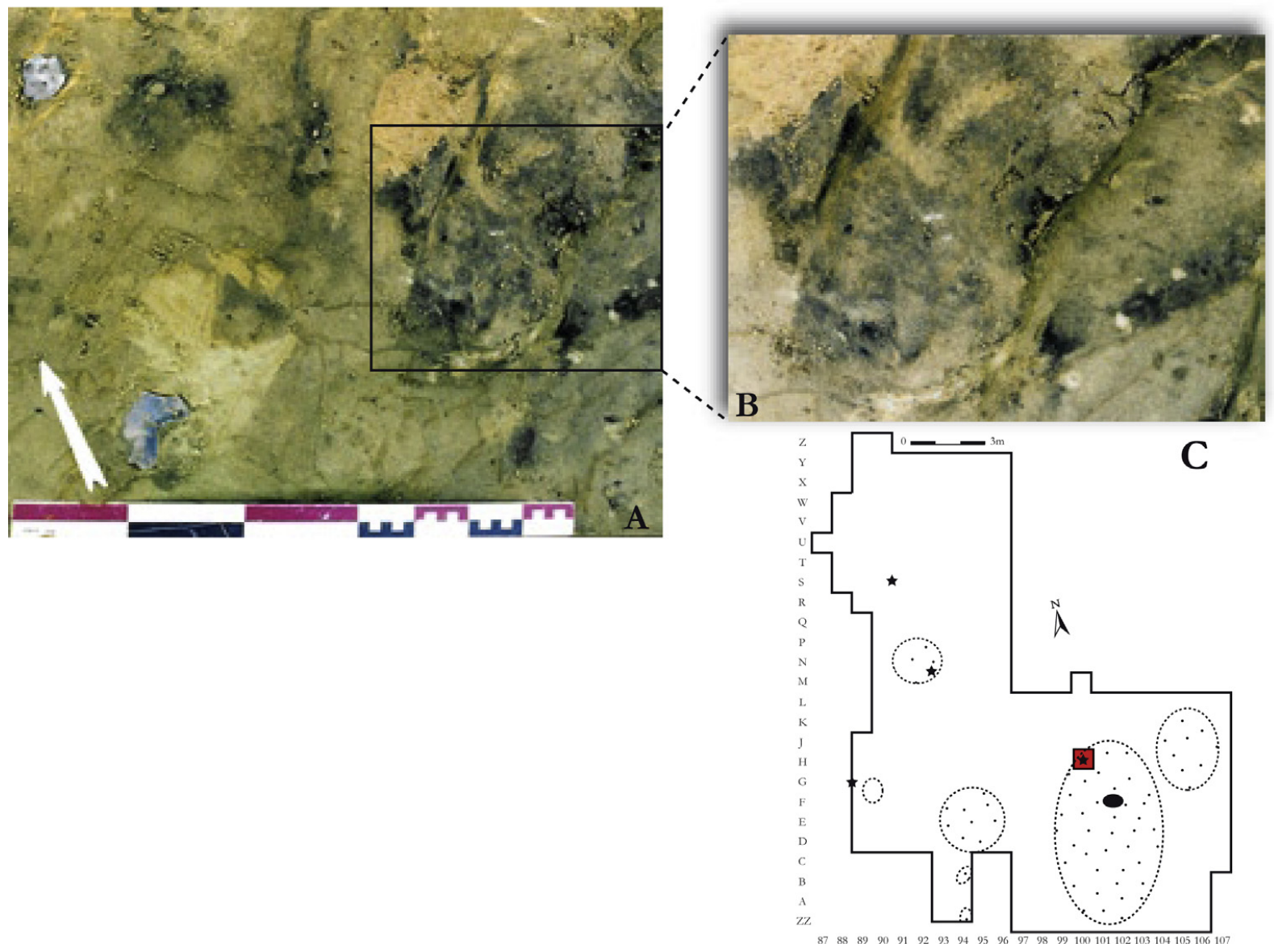

Fig. 6. Niveau N3 de Therdonne. Traces de combustion du carré H100, correspondant à l'échantillon [THECH04]. A. Résidus organiques dont des micro-charbons de bois. B. Zoom sur une des zones charbonneuses. C. Localisation du carré H100.

Level N3 of Therdonne. Combustion traces of square H100, corresponding to sample [THECH04]. A. Organic residues of which wood charcoals. B. Zoom in on one carbonaceous zone. C. Location of square H100.

de type cuvette avait été utilisé pour les foyers, il aurait très difficilement pu être identifié. En effet, des failles de tassement du sédiment sableux ont affecté la structure des zones de combustion.

Il a été possible d'observer sur le terrain que le sable est noirci sur 1 à $2 \mathrm{~cm}$ d'épaisseur. L'impact du foyer semble se limiter à ce liseré noir. Celui de la chauffe, en H100 et F101 situés à proximité immédiate des probables cœurs de foyer (Fig. 6 et 8), n'est pas visible à l'œil nu en coupe. Sans étude micromorphologique, il est impossible de déceler et par conséquent de décrire l'impact de la chauffe sur le sédiment encaissant.

\subsection{Expérimentation d'un foyer in situ}

Suite à la découverte des zones de combustion précédemment décrites, J.-L. Locht décida de tester l'effet d'un foyer sur le sédiment sableux du site pendant la fouille. Cette expérimentation avait pour but de comprendre le type d'enregistrement laissé par un feu dans le sable et l'évolution de sa conservation sur un court laps de temps. À ces fins, un feu fut réalisé in situ sur le sédiment encaissant les niveaux archéologiques. Pour l'allumage, des rafles de maïs couplées à des feuilles de journal furent utilisés (Fig. 10.A). Des os de vache possédant encore de la chair et de la graisse ont servi comme seul et unique combustible (Fig. 10.B et C). La combustion a été 

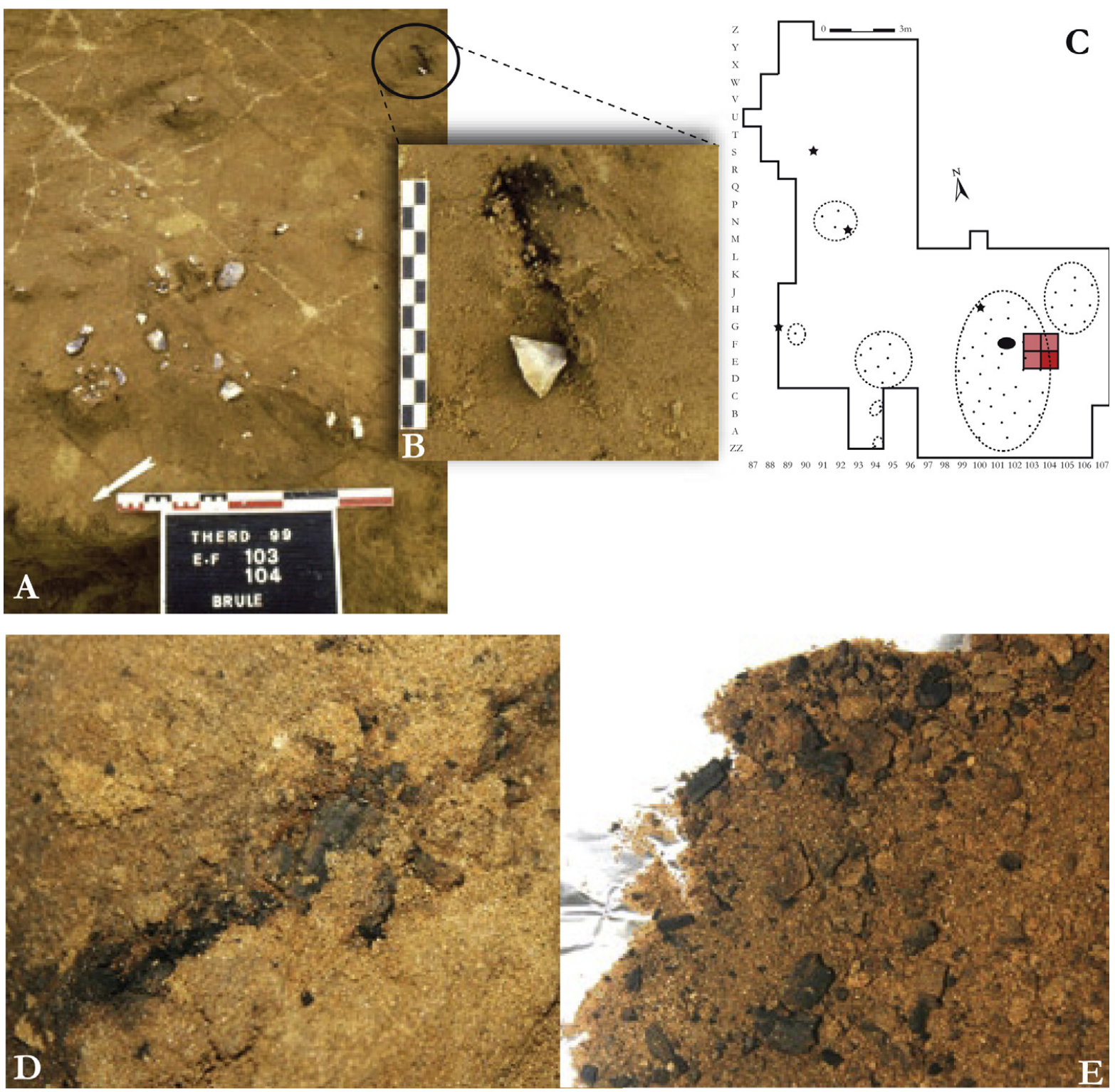

Fig. 7. Niveau N3 de Therdonne. A. Vue des carrés E-F 103-104 en cours de fouilles. B. Zoom sur une zone charbonneuse, carré E104. C. Localisation des mètres carrés E-F 103-104. D. Zoom en laboratoire sur le prélèvement [THECH01 \& 02] de la zone E104. E. Sédiment brûlé et micro-charbons de bois du prélèvement [THECH02] en vrac avant analyse chimique.

Level N3 of Therdonne. A. View of squares E-F 103-104 during the excavation. B. Zoom in on a carbonaceous zone, square E104. C. Location of square-meters E-F 103-104. D. Zoom in at laboratory on the sample [THECH01 \& 02] of the zone E104. E. Burnt sediment and micro-wood charcoals of the sample [THECH02] before chemical analysis.

menée à son terme et le foyer laissé en l'état avant une première série d'observations le lendemain puis une seconde série six mois plus tard.

\subsubsection{Observations jour $j$}

À la fin de la combustion, le jour même, il ne restait à l'emplacement du foyer qu'un amoncellement circulaire de fragments d'os calcinés sur une soixantaine de centimètres de diamètre pour une dizaine de centimètres d'épaisseur (Fig 10D et 11A et C). Une tâche noirâtre qui s'est étendue progressivement en périphérie du foyer lors de la combustion était bien visible (Fig. 10.B, D). Cette tâche était probablement constituée par les graisses liquéfiées. 

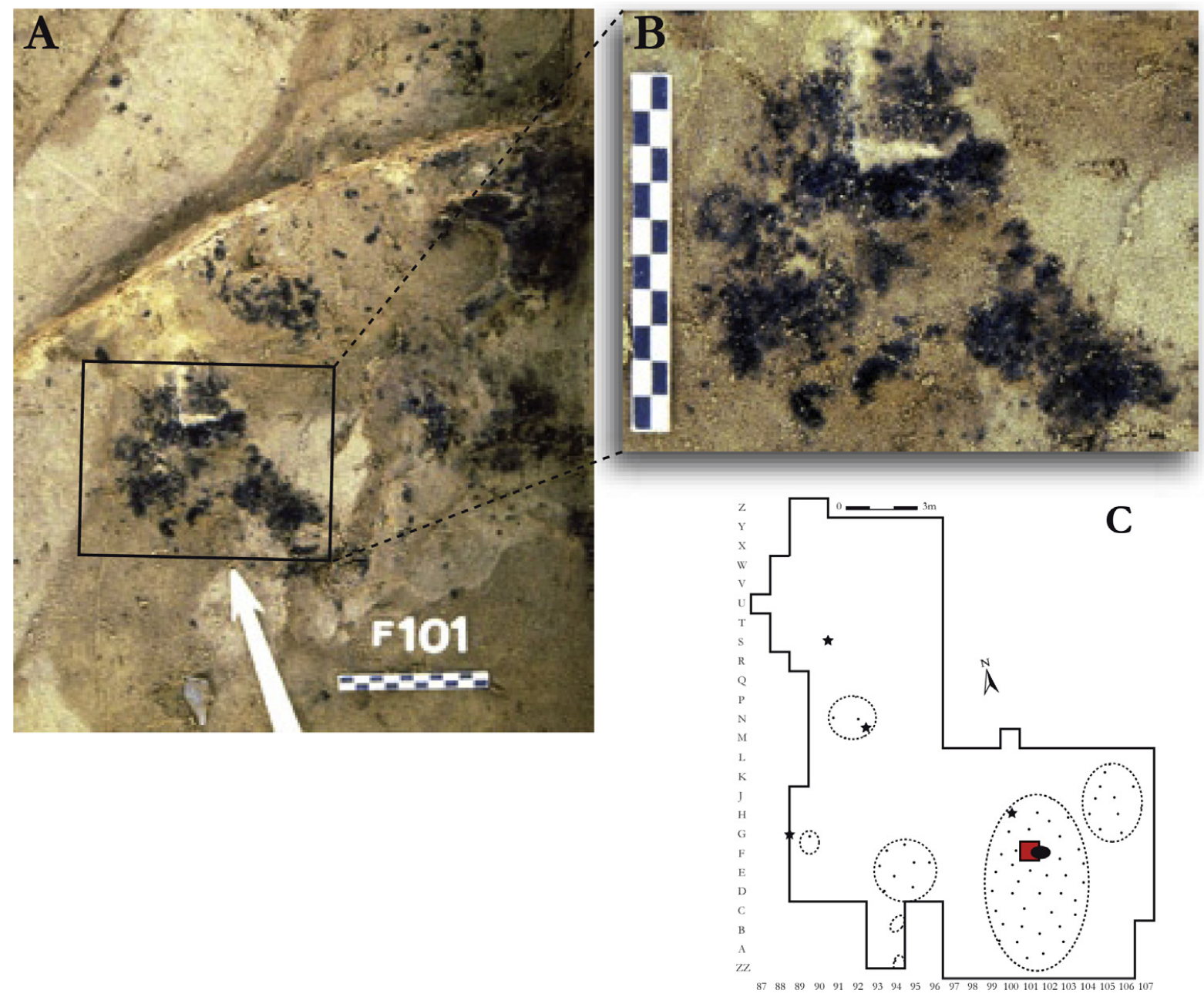

Fig. 8. Niveau N3 de Therdonne. Traces de combustion du carré F101, correspondant à l'échantillon [THECH05]. A. Résidus organiques dont des micro-charbons de bois. B. Zoom sur une des zones charbonneuses. C. Localisation du carré F101.

Level N3 of Therdonne. Combustion traces of square F101, corresponding to sample [THECH05]. A. Organic residues of which wood charcoals. B. Zoom in on one carbonaceous zone. C. Location of square F101.

\subsubsection{Observations jour $j+1$}

Le lendemain, après une nuit de pluie, de nouvelles observations furent pratiquées. La pluie a totalement lessivé la tâche de graisse pourtant bien visible la veille (Fig. 10.B, D et Fig. 11). Sous la couche de fragments d'os calcinés, s'était développée une couche cendreuse noirâtre discontinue à la surface du sédiment de forme globalement circulaire, aux contours flous (Fig. 11A). En coupe, au milieu du foyer, le sédiment a visiblement été altéré sur une épaisseur de 1 à $5 \mathrm{~cm}$ (Fig. $11 \mathrm{C}, \mathrm{D})$. Une couche noirâtre $(5 \mathrm{Y} 2.5 / 1)$ de 1 à $3 \mathrm{~cm}$ de puissance coiffait l'ensemble. Une couche intermédiaire marron jaune (10YR4/3) de 1 à $2 \mathrm{~cm}$ se dessinait par endroit. Sous cet ensemble, le sédiment jaune (10YR7/6) ne semblait pas avoir subi d'altérations visibles à l'œil nu. Lors de la réalisation de la coupe, des blocs du foyer furent prélevés en vue de futures analyses.

\subsubsection{Observations $j+200$}

Après six mois d'exposition aux aléas climatiques, le foyer n'a pas perdu globalement sa forme circulaire. Cependant, ses limites étaient moins nettes suite au lessivage à répétition. Le 

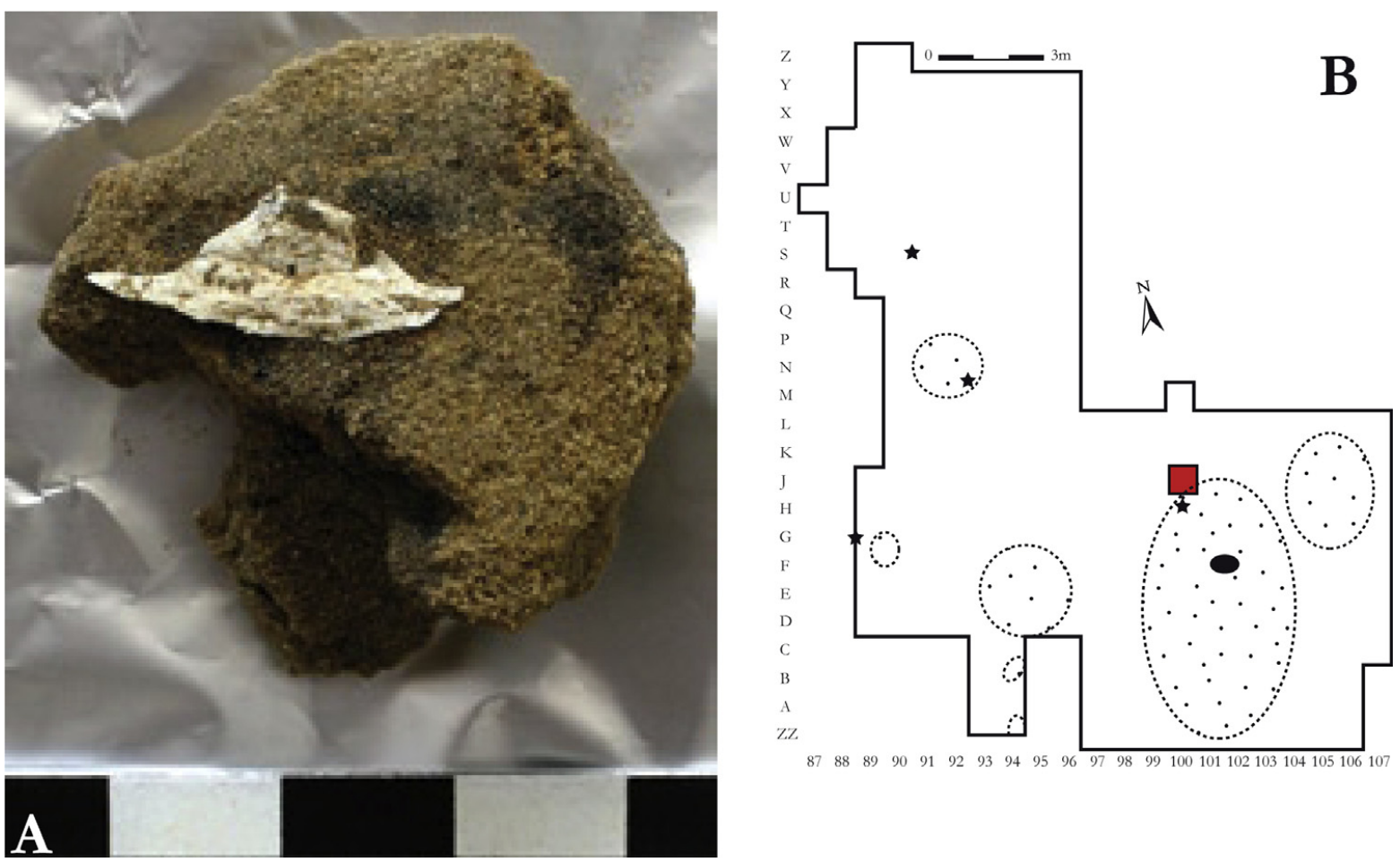

Fig. 9. Niveau N3 de Therdonne. A. Fragment d'os brûlé retrouvé dans l'échantillon [THECH09] du carré j100. B. Localisation du carré $\mathrm{j} 100$.

Level N3 of Therdonne. A. Fragment of burnt bone found in sample [THECH09] of square j100. B. Location of square $j 100$.

vent notamment a entraîné une dispersion voire une disparition de la couche de fragments d'os calcinés (Fig. 11E).

\subsubsection{Estimation de la température de chauffe par thermoluminescence}

Un test de thermoluminescence a été effectué par N. Debenham sur un prélèvement de sédiment du foyer expérimental afin d'estimer la température de chauffe qu'a subi le sédiment. L'échantillon de sable analysé est situé $1 \mathrm{~cm}$ sous la surface du foyer. La thermoluminescence indique à $1 \mathrm{~cm}$ de profondeur que le sédiment a été chauffé à environ $250{ }^{\circ} \mathrm{C}$.

\subsection{Résultats de l'étude des silex et des calcaires brûlés}

Une part conséquente du matériel lithique du niveau N3 a subi l'action du feu. En effet, 3333 silex taillés présentent des traces de thermoaltération par le feu, soit plus de $7 \%$ de l'industrie (Tableau 11). La distribution par catégorie technologique des artefacts brûlés est globalement calquée sur celle des éléments non brûlés, exception faite des petits éléments. Les esquilles et les éclats de moins de $3 \mathrm{~cm}$ brûlés représentent respectivement $7 \%$ et $16 \%$ de leurs catégories tandis que les pièces brûlées représentent entre 1 et $3 \%$ pour les autres catégories. La fraction fine de l'assemblage a donc été exposée au feu de manière privilégiée.

Sur une échelle graduelle des altérations thermiques allant de 1 à 4 , les silex brûlés sont très majoritairement fortement altérés par le feu (Fig. 12 et 13). Ceci prouve qu'une grande partie des silex brûlés retrouvés l'ont été directement dans le foyer ou à proximité immédiate du cœur de chauffe. 


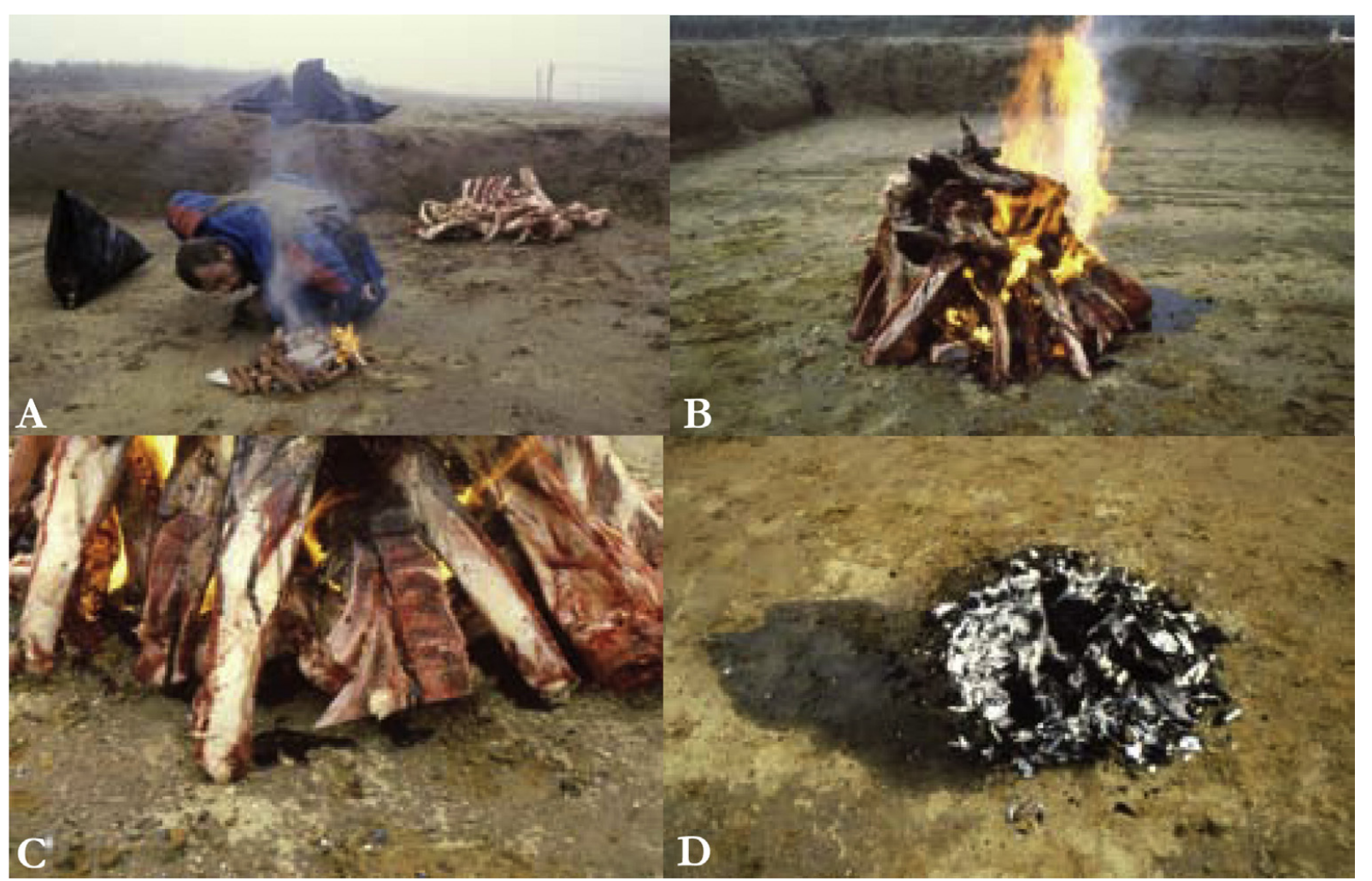

Fig. 10. Expérimentation de foyer à Therdonne. A. Allumage du feu avec des rafles de maïs et du papier journal. Au second plan, ossements de vache servant de combustible. B. Combustion en cours des os partiellement décharnés. À droite, début de l'extension d'une tâche de graisses liquéfiées. C. Vue de détail du foyer au début de la combustion des os. D. Le foyer juste après l'arrêt de la combustion. À gauche, tâche étendue de graisses liquéfiées.

Experimental fire at Therdonne. A. Lighting of fire with corn cobs and newspaper. In the background, bones of cow used as fuel. B. Ongoing fire of partially emaciated bones. On the right, beginning of the expansion of a liquified fat task. $C$. Detailed view of the fire at the beginning of the combustion of bones. D. Fire just after the end of the combustion. On the left, expansed task of liquified fat.

Sept blocs calcaires blancs riches en tests à l'œil nu ont été découverts dans le niveau N3 (Fig. 14). Ces blocs étudiés en lames minces par D. Vachard comprennent plusieurs familles de foraminifères : Miliole Quinqueloculininae, Orbiltolite Complanatus, Miliole Pyrgo, Miliole Triloculina (Fig. 15). Ce cortège de foraminifères permet une attribution de ces calcaires au Lutétien moyen. A. Blondeau et al. dénomment ces calcaires Banc royal, décrits comme « fin, zoogène, riche en Miliolidae et Orbitolites complanatus » (Blondeau et al., 1970, p. 8). Le calcaire lutétien moyen «affleure sur le plateau, de part et d'autre de la vallée du Thérain, sur le plateau de Cambronne-lès-Clermont et d'Auvillers, ainsi que dans la région de Liancourt » (Blondeau et al., 1970, p. 8). L'affleurement en place le plus proche se situe sur la rive opposée du Thérain à $3 \mathrm{~km}$ au Sud du gisement (Fig. 16). Les arêtes émoussées des blocs mis au jour indiquent qu'ils ont été recueillis en position secondaire. Ils ont dû être ramassés aux pieds des buttes tertiaires le long du Thérain. À l'image d'un bloc de grès auversien parallélipédique mis au jour dans le même niveau (Hérisson, 2012, p. 127-128), ces calcaires démontrent un passage des Néandertaliens dans la région sud-est de Therdonne avant leur abandon sur le gisement. Trois blocs de calcaire du Lutétien moyen sur sept possèdent des stigmates d'altération thermique par le feu (Tableau 12, Fig. 17). Une à trois de leurs faces arbore une oxydation rouge ainsi que des négatifs de cupules thermiques (mudcracks) dans le cas du bloc le plus altéré (Fig. 17). Au moins trois blocs calcaires ont donc subi une altération thermique due à leur proximité immédiate des 

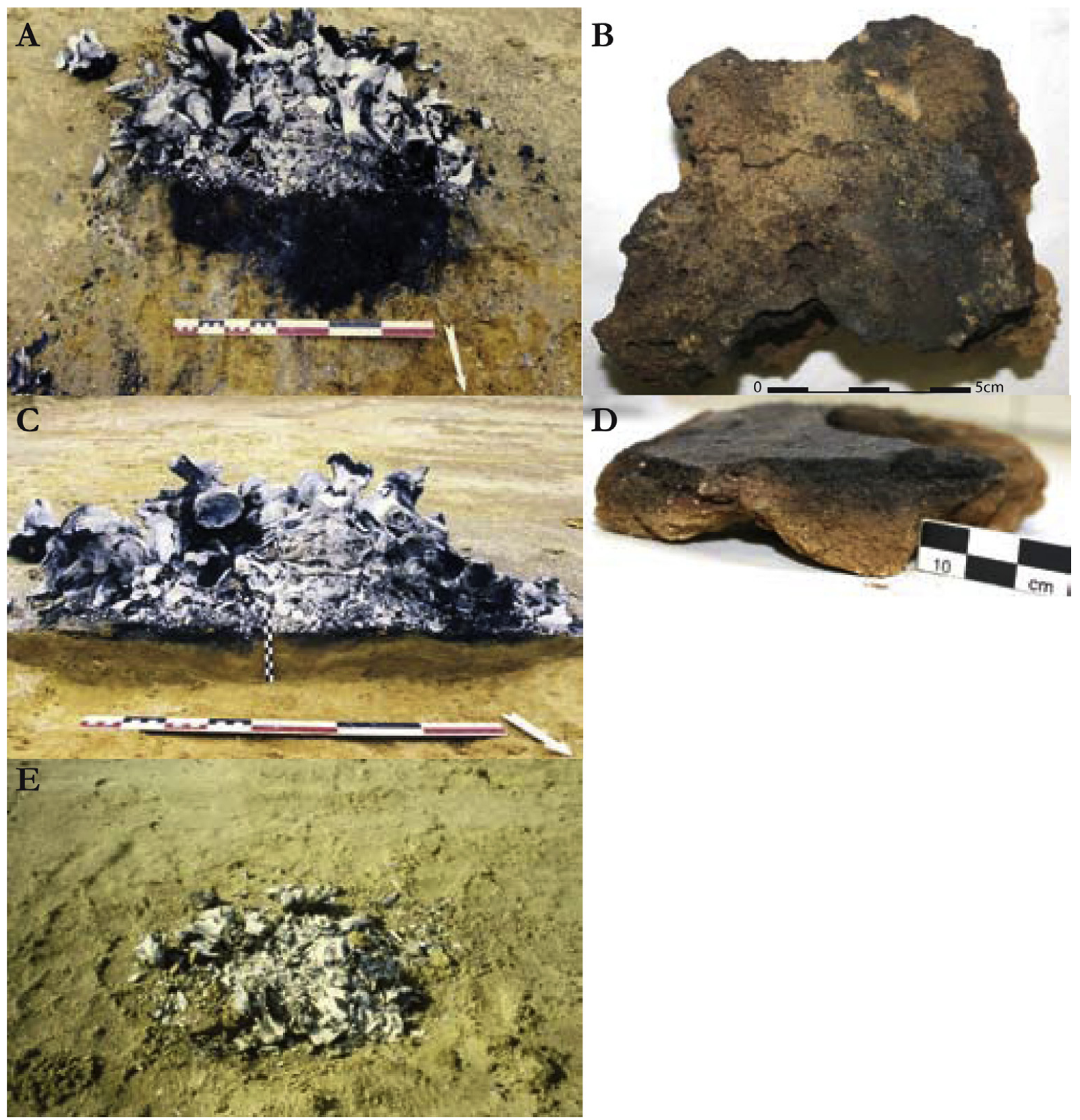

Fig. 11. Résidus de l'expérimentation de foyer à Therdonne. A. Foyer après combustion dont la moitié sud a été nettoyée des charbons et cendres superficielles afin d'observer l'impact de la combustion sur le substrat. B. Prélèvement du foyer expérimental vu de dessus sur lequel peut être observé la présence de petites cupules circulaires en creux qui se sont formées sous l'effet de la chaleur et un film luisant noir organique résultant de la combustion partielle des graisses. C. Vue en coupe du foyer après prélèvement de la partie méridionale sur laquelle se distingue nettement la modification et l'enrichissement en matière organique de la couche superficielle du substrat, impactée sur près de $3 \mathrm{~cm}$ par endroit. D. Vue en coupe d'un prélèvement de la partie méridionale du foyer sur lequel la transformation graduelle du sédiment à cause de la chauffe et de la combustion est bien visible. E. Vue de l'état du foyer expérimental livré aux intempéries six mois durant. Residues of the experimental fire at Therdonne. A. Hearth after combustion with the southern part cleaned superficial charcoals and ashes off for observing impact of the combustion on the substratum. B. Top view of the sample of the experimental fire on which can be observed the presence of little circular hollow ice cupules formed under the heating effect and a gleaming black film resulting from partial combustion of fat. C. After sampling of the Southern part of the hearth, section view on which the modification and the enrichment in organic matter of the superficial layer of the substratum, impacted on about $3 \mathrm{~cm}$ deep by place. D. Section view of a sample of the Southern part of the hearth on which the gradual transformation of the sediment because of the heating and of the combustion is very visible. E. View of state of the experimental hearth after six months of weathering. 
Tableau 10

Niveau D de Biache-Saint-Vaast. Décompte et pourcentage des différentes catégories technologiques d'artefacts lithiques brûlés et non brûlés.

Level D of Biache-Saint-Vaast. Counting and percentage of different technological categories of burnt and non-burnt lithic artifacts.

\begin{tabular}{lllcl}
\hline Catégories & NR brûlés & $\begin{array}{l}\% \text { du total du } \\
\text { NR brûlés }\end{array}$ & $\begin{array}{l}\text { NR total } \\
\text { (brûlés et non brûlés) }\end{array}$ & $\begin{array}{l}\% \text { du NR brûlés } \\
\text { de chaque catégorie }\end{array}$ \\
\hline Éclats Levallois & 0 & - & 18 & - \\
Pointes Levallois & 0 & - & 0 & - \\
Éclats Kombewa & 0 & - & 1 & - \\
Éclats non corticaux & 2 & $40 \%$ & 70 & $2,9 \%$ \\
Éclats semi-corticaux & 1 & $20 \%$ & 37 & $2,7 \%$ \\
Éclats corticaux & 0 & - & 20 & - \\
Entame & 0 & - & 0 & - \\
Éclats caractéristiques & 0 & - & 7 & - \\
Nucléus & 0 & - & 10 & - \\
Cassons & 0 & - & 5 & - \\
Cassons gélivés & 0 & - & 0 & $0,4 \%$ \\
Éclats $<3$ cm & 1 & $20 \%$ & 760 & $1,3 \%$ \\
Esquilles & 1 & $20 \%$ & 3 & - \\
Blocs testés & 0 & - & 0 & - \\
Percuteurs & 0 & $100 \%$ & 506 & $1,0 \%$ \\
Total & 5 & & &
\end{tabular}

\section{Tableau 11}

Niveau N3 de Therdonne. Décompte et pourcentage des différentes catégories technologiques d'artefacts lithiques brûlés et non brûlés.

Level N3 of Therdonne. Counting and percentage of different technological categories of burnt and non-burnt lithic artifacts.

\begin{tabular}{|c|c|c|c|c|c|}
\hline Catégories & $\begin{array}{l}\text { NR } \\
\text { brûlés }\end{array}$ & $\begin{array}{l}\% \text { du total } \\
\text { du NR brûlés }\end{array}$ & $\begin{array}{l}\% \text { du total du NR } \\
\text { brûlés ss }<3 \mathrm{~cm}\end{array}$ & $\begin{array}{l}\text { NR total } \\
\text { (brûlés et non brûlés) }\end{array}$ & $\begin{array}{l}\% \text { du NR brûlés de } \\
\text { chaque catégorie }\end{array}$ \\
\hline Éclats Levallois & 8 & $0,2 \%$ & $5,6 \%$ & 492 & $1,6 \%$ \\
\hline Pointes Levallois & 3 & $0,1 \%$ & $2,1 \%$ & 138 & $2,2 \%$ \\
\hline Éclats Kombewa & 0 & - & - & 4 & - \\
\hline Éclats discoïdes & 1 & - & $0,7 \%$ & 35 & $2,9 \%$ \\
\hline Éclats type Pucheuil & 0 & - & - & 19 & - \\
\hline Lames & 0 & - & - & 3 & - \\
\hline Éclats non corticaux & 80 & $2,4 \%$ & $55,6 \%$ & 2418 & $3,3 \%$ \\
\hline Éclats semi-corticaux & 24 & $0,7 \%$ & $16,7 \%$ & 981 & $2,4 \%$ \\
\hline Éclats corticaux & 25 & $0,8 \%$ & $17,4 \%$ & 700 & $3,6 \%$ \\
\hline Entame & 1 & - & $0,7 \%$ & 40 & $2,5 \%$ \\
\hline Éclats caractéristiques & 1 & - & $0,7 \%$ & 92 & $1,1 \%$ \\
\hline Nucléus & 1 & - & $0,7 \%$ & 87 & $1,1 \%$ \\
\hline Cassons & 0 & - & - & 142 & - \\
\hline Cassons gélivés & 0 & - & - & 0 & - \\
\hline Éclats $<3 \mathrm{~cm}$ & 754 & $22,6 \%$ & l & 4837 & $15,6 \%$ \\
\hline Esquilles & 2435 & $73,1 \%$ & l & 36174 & $6,7 \%$ \\
\hline Blocs testés & 0 & - & - & 0 & - \\
\hline Percuteurs & 0 & - & - & 1 & - \\
\hline Total & 3333 & $100 \%$ & $100 \%$ & 46163 & $7,2 \%$ \\
\hline
\end{tabular}




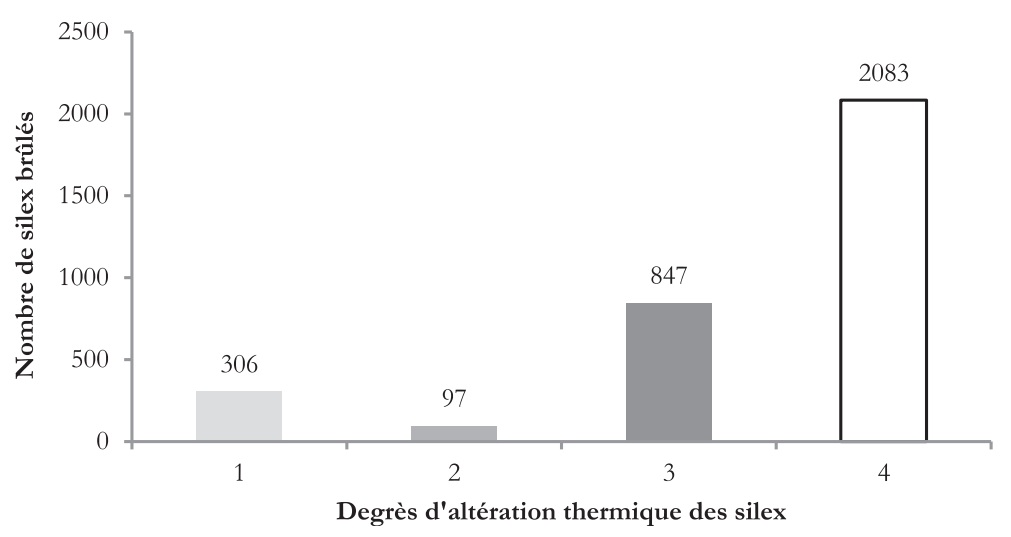

Fig. 12. Niveau N3 de Therdonne. Distribution des silex brûlés en quatre catégories de thermoaltération croissante. Level N3 of Therdonne. Distribution of burnt flint in four categories of growing thermoalteration.

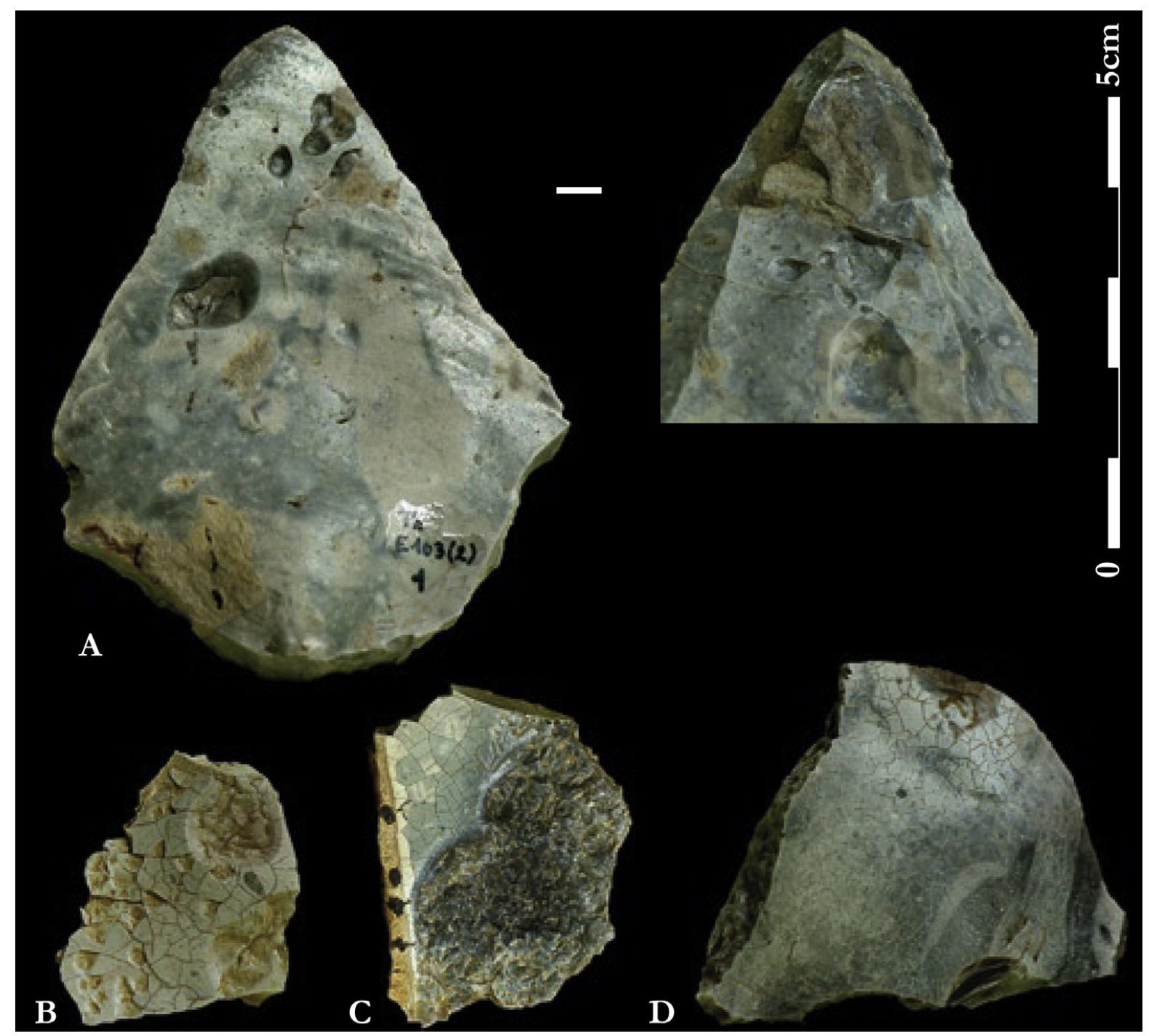

Fig. 13. Niveau N3 de Therdonne. A. Pointe Levallois brûlée en partie distale. B-D. Fragments de silex brûlés. Level N3 of Therdonne. A. Levallois point burnt on the distal part. B-D. Burnt flint fragments. 


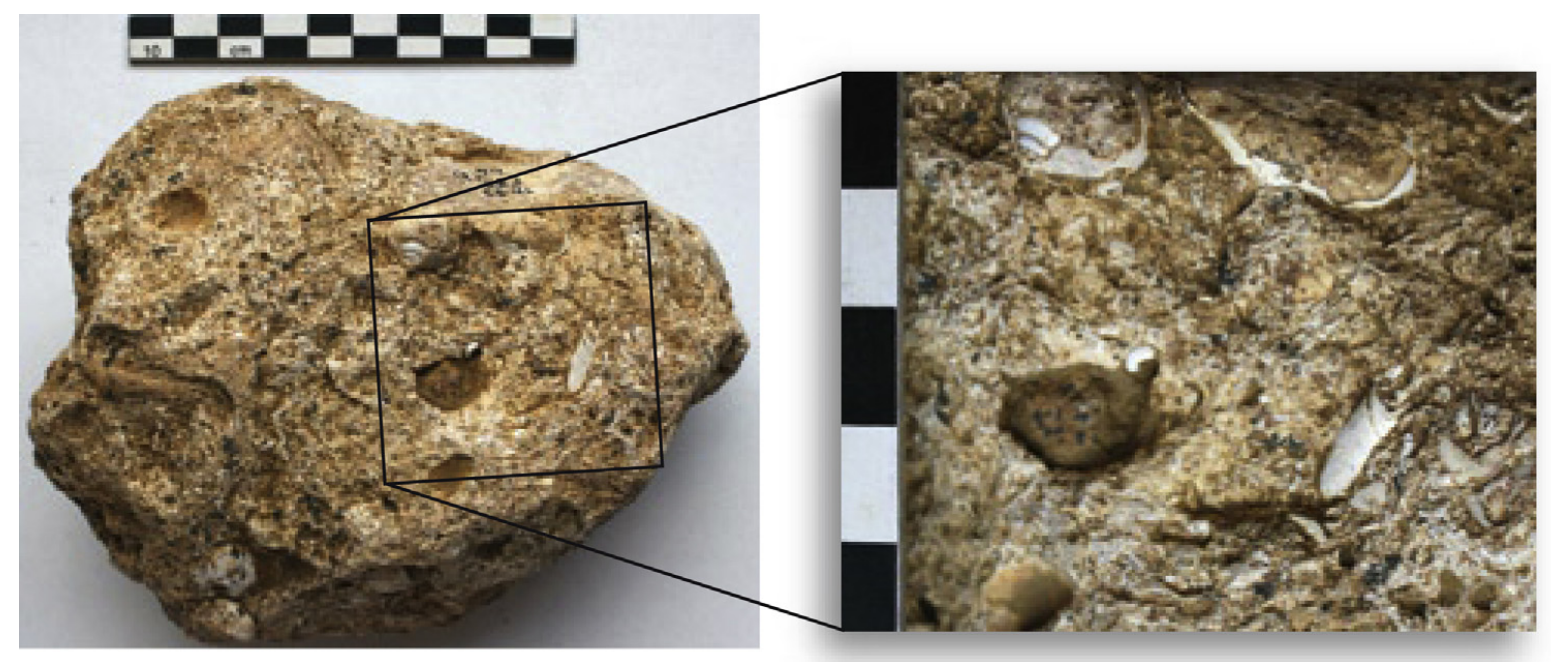

Fig. 14. Niveau N3 de Therdonne. Bloc de calcaire du Lutétien moyen. Level N3 of Therdonne. Middle Lutetain block of limestone.
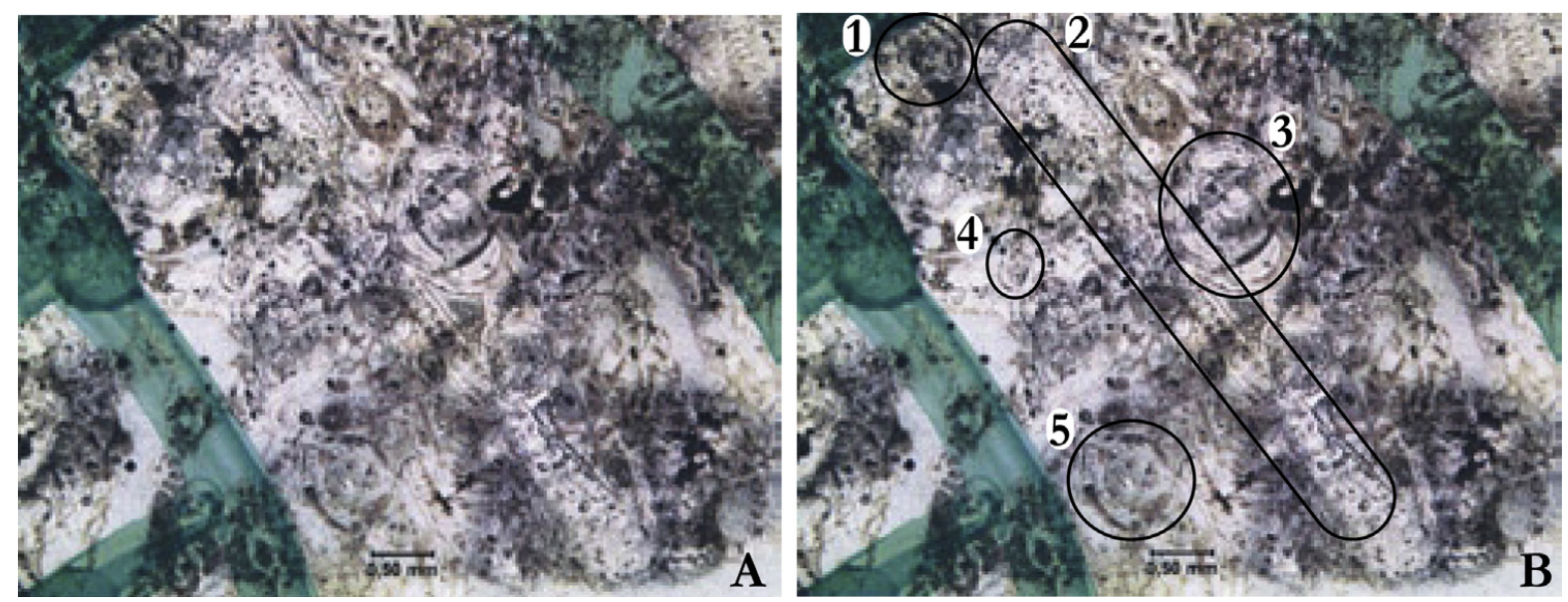

Fig. 15. Niveau N3 de Therdonne. Lame mince issu du bloc calcaire X 91 (2). B. Détermination des foraminifères par D. Vachard, 1 - Miliole Quinqueloculininae ; 2 - Orbiltolite Complanatus ; 3 - Miliole Pyrgo ; 4 - Miliole Triloculina ; 5 - Miliole Pyrgo. Cliché : D. Vachard.

Level N3 of Therdonne. Thin section of the limestone block X 91 (2). B. Determination of foraminiferas by D. Vachard, 1 Miliole Quinqueloculininae; 2 - Orbiltolite Complanatus; 3 -Miliole Pyrgo; 4 -Miliole Triloculina; 5 - Miliole Pyrgo. Cliché: D. Vachard.

foyers lors de la combustion. Il paraît peu probable que ces éléments aient servi à former un foyer construit eu égard à leurs dimensions, leur faible nombre et leur distribution éparse sur la zone fouillée. Cependant, leur localisation à proximité des zones hypothétiques de combustion (Fig. 18 et 19) ainsi que les stigmates thermiques visibles laissent envisager leur utilisation dans le cadre d'activité(s) intimement liée(s) à l'utilisation du feu. Le lieu de leur récolte implique une anticipation des besoins et un import volontaire programmé sur le site. Ce type d'outils devait jouer un rôle important dans les chaînes opératoires mises en œuvre, peut-être dans le domaine alimentaire.

\subsection{Principaux résultats de l'analyse chimique}

Les résultats des analyses chimiques menées sur les sept échantillons archéologiques convergent vers une interprétation unique des zones de combustion mises au jour (Hérisson, 


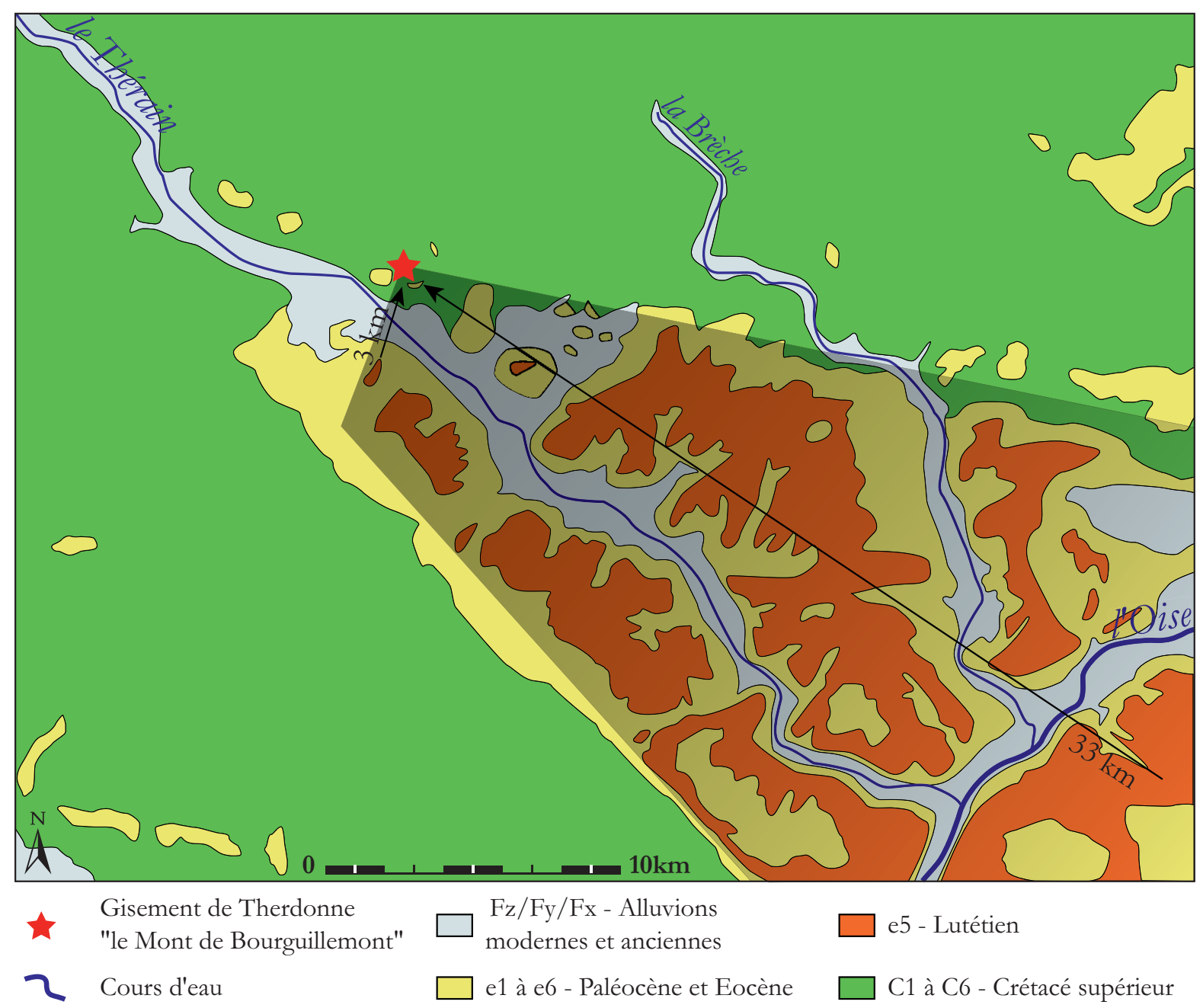

Fig. 16. Carte de localisation des affleurements actuels du Lutétien aux alentours du gisement de Therdonne (extrait de la carte géologique d'après Blondeau et al., 1970).

Location map of current lutetian outcrops around the site of Therdonne (extract of the geological map after Blondeau et al., 1970).

2012). Les zones noires observées lors de la fouille proviennent de la combustion de matière organique par l'Homme. De la matière végétale a été utilisée comme combustible comme le montre entre autres la distribution des alcanes retrouvés. La présence du rétène, de l'acide déhydroabiétique et d'autres hydrocarbures aromatiques polycycliques indiquent que le bois brûlé est issu de la famille des conifères. Plus précisément, il est très probable que l'essence soit du pin (Pinus) eu égard à l'environnement steppique reconstitué et à la plus proche séquence pollinique connue (séquence de Biache-Saint-Vaast par Munaut, 1988). De manière plus spéculative, il est possible de concevoir un approvisionnement de proximité en bois, provenant d'un bosquet de pins coiffant la butte tertiaire du mont de Bourguillemont. La question de l'utilisation de l'os comme deuxième combustible reste ouverte. En effet, seuls quelques petits fragments d'os brûlés ont été retrouvés, à l'image de celui qui était présent dans l'échantillon [THECH09] (Fig. 9). Les amides identifiés peuvent aussi indiquer une dégradation thermique de l'os. Les potentiels restes osseux brûlés ont pu disparaître sous l'action de l'acidité du sol et subir le même sort que les restes osseux non brûlés, la disparition (non conservation). Tous ces 
Tableau 12

Niveau N3 de Therdonne. Caractéristiques des roches calcaires du Lutétien moyen brûlées et non brûlées. Level N3 of Therdonne. Charateristics of burnt and non-burnt limestones of Middle Lutetian.

\begin{tabular}{|c|c|c|c|c|c|c|c|}
\hline Référence & $\begin{array}{l}\text { Longueur } \\
(\mathrm{mm})\end{array}$ & $\begin{array}{l}\text { Largeur } \\
(\mathrm{mm})\end{array}$ & $\begin{array}{l}\text { Épaisseur } \\
(\mathrm{mm})\end{array}$ & $\begin{array}{l}\text { Masse } \\
(\mathrm{g})\end{array}$ & Matière première & $\begin{array}{l}\text { Degrés d'altération } \\
\text { thermique }\end{array}$ & $\begin{array}{l}\text { Nombre de } \\
\text { faces oxydées }\end{array}$ \\
\hline F89 (3) 44 & 148 & 122 & 81 & 1010 & Calcaire lutétien moyen & Néant & 0 \\
\hline J89 51 & 62 & 51 & 26 & 66 & Calcaire lutétien moyen & Néant & 0 \\
\hline D105 & 70 & 37 & 17 & 28 & Calcaire lutétien moyen & Néant & 0 \\
\hline X91 (2) & 152 & 111 & 52 & 454 & Calcaire lutétien moyen & Néant & 0 \\
\hline H91 63 & 100 & 74 & 65 & 346 & Calcaire lutétien moyen & $\begin{array}{l}\text { Légère oxydation } \\
\text { rouge }\end{array}$ & 1 \\
\hline K99 4 & 66 & 52 & 55 & 104 & Calcaire lutétien moyen & Oxydation rouge & 2 \\
\hline F92 30 & 66 & 64 & 41 & 123 & Calcaire lutétien moyen & $\begin{array}{l}\text { Oxydation rouge et } \\
\text { blanche + cupules } \\
\text { thermiques }\end{array}$ & 3 \\
\hline
\end{tabular}

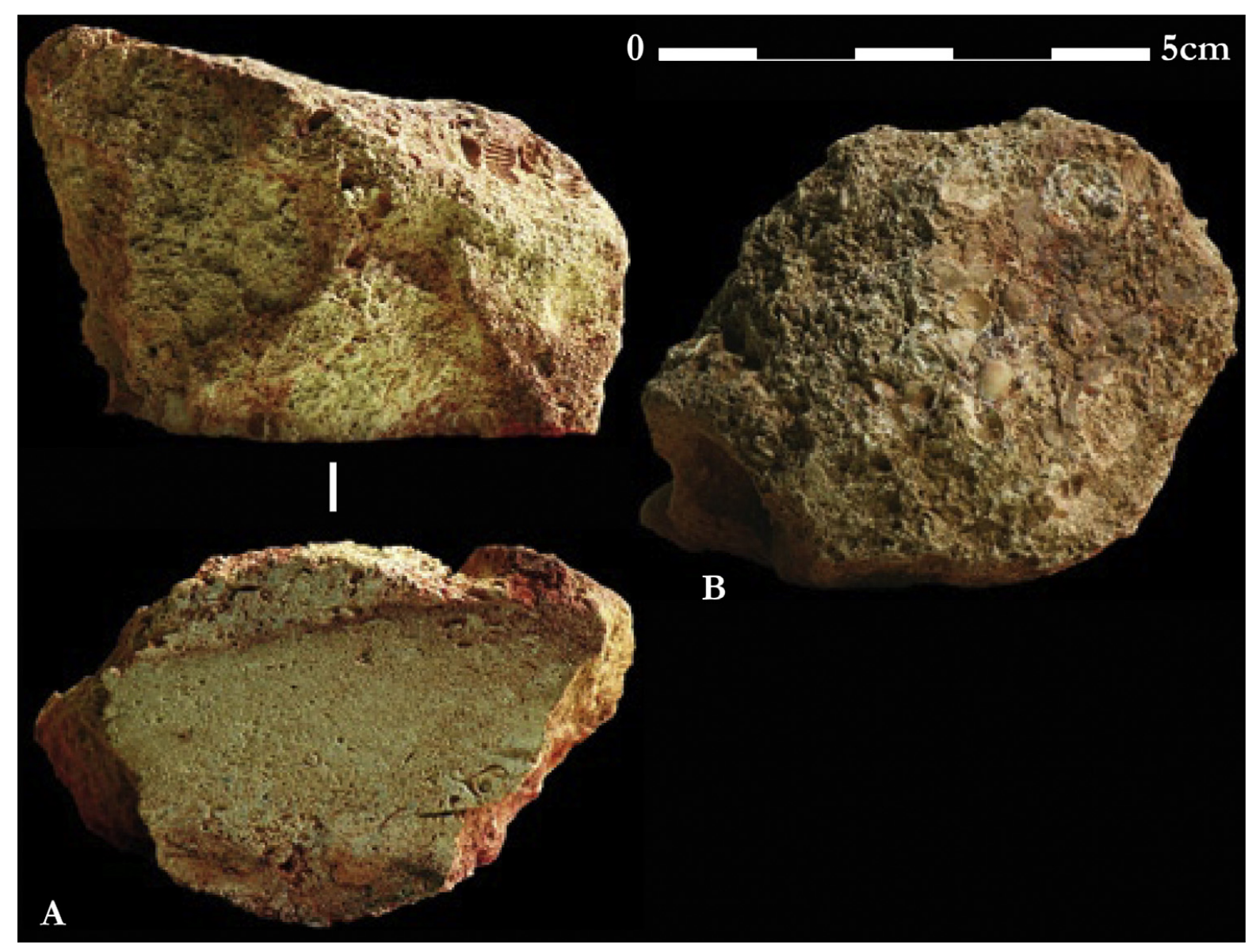

Fig. 17. Blocs de calcaire lutétien moyen affectés par le feu, F92 30 (A) et K99 4 (B). A. Négatifs de cupules thermiques (mudcracks) visibles en surface en haut et impact de la chauffe en profondeur par oxydation visible en section. B. Oxydation due à la chauffe.

Burnt limestone blocks, F92 30 (A) and K99 4 (B). A. Negatives of thermic cupules (mudcracks) visible on the surface at the top and deeply heating impact by oxidisation visible in section. B. Oxidisation due to heating. 
éléments ne permettent pas d'exclure ni d'affirmer l'utilisation d'os comme combustible. Cependant, les éléments jusqu'à présent réunis plaident en faveur d'une absence d'utilisation d'os comme combustible.

Si la question de la contribution animale en tant que combustible ne peut être tranchée, celle de la présence de molécules d'origine animale dans les foyers ne laisse aucun doute. La distribution des acides gras saturés et des $n$-alcanes, la présence des acides dicarboxyliques, de cholestérol, de méthyles cétones et aldéhydes courts, des lactones et des alcools gras sont des preuves de la cuisson de viande dans les foyers de Therdonne. Les profils des acides gras saturés et des $n$-alcanes, entre autres, plaident en faveur de viande de ruminant et plus précisément de la famille des bovidés. Cet élément est en adéquation avec les seuls restes fauniques préservés et retrouvés sur le gisement, trois dents de bovidés.

Quant aux différenciations entre échantillons et entre zones ou foyers de combustion distincts, l'analyse n'a en l'état actuel pas permis de mettre en évidence des éléments suffisamment probants pour attester de fonctions ou de modes de fonctionnement différents. Seul l'échantillon [THECH04] semble se différencier par la présence d'acides gras isoprénoïdes, sans que cela permette d'affirmer de manière certaine la présence de graisse de poisson dans cet échantillon.

L'apport de l'analyse chimique dans l'étude des foyers de Therdonne est conséquent. Ces données doivent être réunies et spatialisées avec les éléments précédemment recueillis afin de proposer des réponses aux questions que posaient ces foyers lors de leur découverte.

\section{7. À la recherche de l'emplacement des foyers}

L'existence de structures de combustion est indéniable à Therdonne. Les résidus organiques dont des charbons de bois et d'os brûlés, les pièces lithiques brûlées et les calcaires brûlés témoignent de l'usage du feu sur le site. Plus complexe est la localisation exacte du cœur du foyer puisqu'aucune structure apparente n'a été retrouvée lors de la fouille. En effet, l'aménagement se résumait au maximum à un creusement superficiel dans le sable, impossible à déceler en fouille dans le substrat sableux. Si l'emplacement du cœur de chauffe n'est pas directement identifiable sur le terrain, la mise en corrélation de l'ensemble des témoins de la chauffe permet d'avancer des hypothèses quant à la localisation des foyers. Ainsi, l'analyse des plans de répartition des éléments brûlés (Fig. 18A-C) mis en parallèle avec la localisation des zones de charbons et de résidus organiques observés lors de la fouille (Fig. 5), permet de définir 12 zones potentielles de localisation de foyer (Fig. 19). L'emplacement d'au moins cinq foyers sur l'aire de fouille est un élément quasi-certain eu égard au nombre de preuves convergentes pour ces zones. Quatre zones pourraient avoir accueilli des foyers vu les témoins convaincants attestant de cette présence. Enfin, deux zones peuvent témoigner de l'existence de foyer en ces localisations, d'après quelques indices comme le pourcentage de pièces lithiques brûlées. Même si les seules zones certaines sont retenues comme ayant effectivement abrité des foyers, la surface explorée par la fouille se retrouve régulièrement ponctuée de structure de combustion. Ces structures se situent toutes en périphérie immédiate (entre 0 et $2 \mathrm{~m}$ ) des zones de forte concentration lithique (Fig. 18D). Seule une zone de combustion localisée de manière fiable se retrouve hors des zones de forte concentration du matériel. Si le scénario optimiste prenant en compte l'ensemble des hypothétiques zones de foyers est considéré, cela donnerait un ratio d'un foyer tous les $25 \mathrm{~m}^{2}$, phénomène étonnant, voir déroutant pour une occupation du Paléolithique moyen ancien et en plein air. 

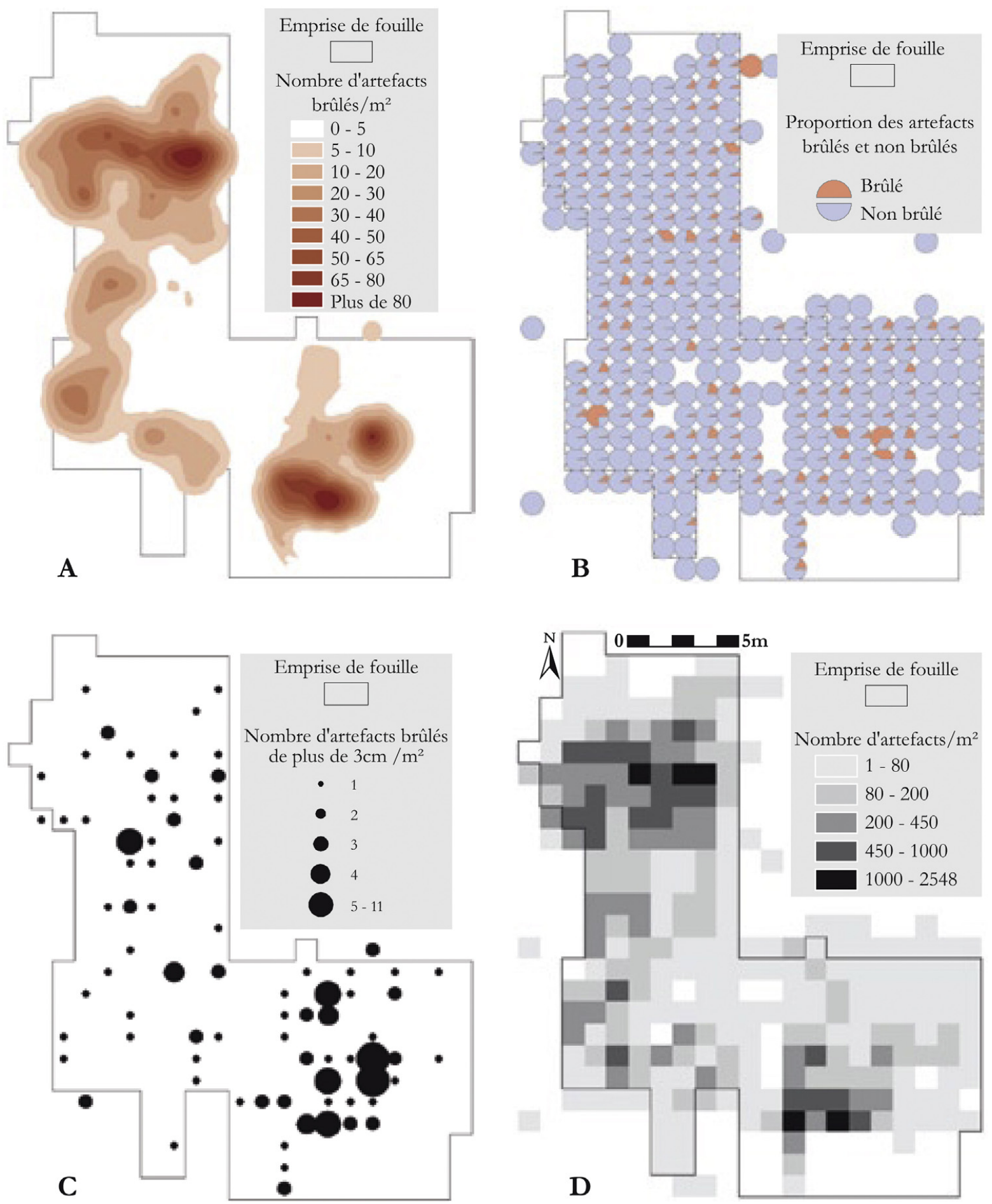

Fig. 18. Niveau N3 de Therdonne. A. Plan d'isodensité des silex brûlés par mètre carré. B. Répartition par pourcentage des silex brûlés et non brûlés par mètre carré. C. Répartition ponctuelle des silex brûlés en excluant les esquilles et les éclats de moins de $3 \mathrm{~cm}$. D. Plan de densité par mètre carré de l'ensemble des artefacts lithiques.

Level N3 of Therdonne. A. Isodensity map of burnt flint per square meter. B. Ditribution per percentage of burnt and nonburnt flint per square meter. C. Pointwise distribution of burnt flint excluding flakes of less than $3 \mathrm{~cm}$. D. Density map per square meter of all lithic artifacts. 

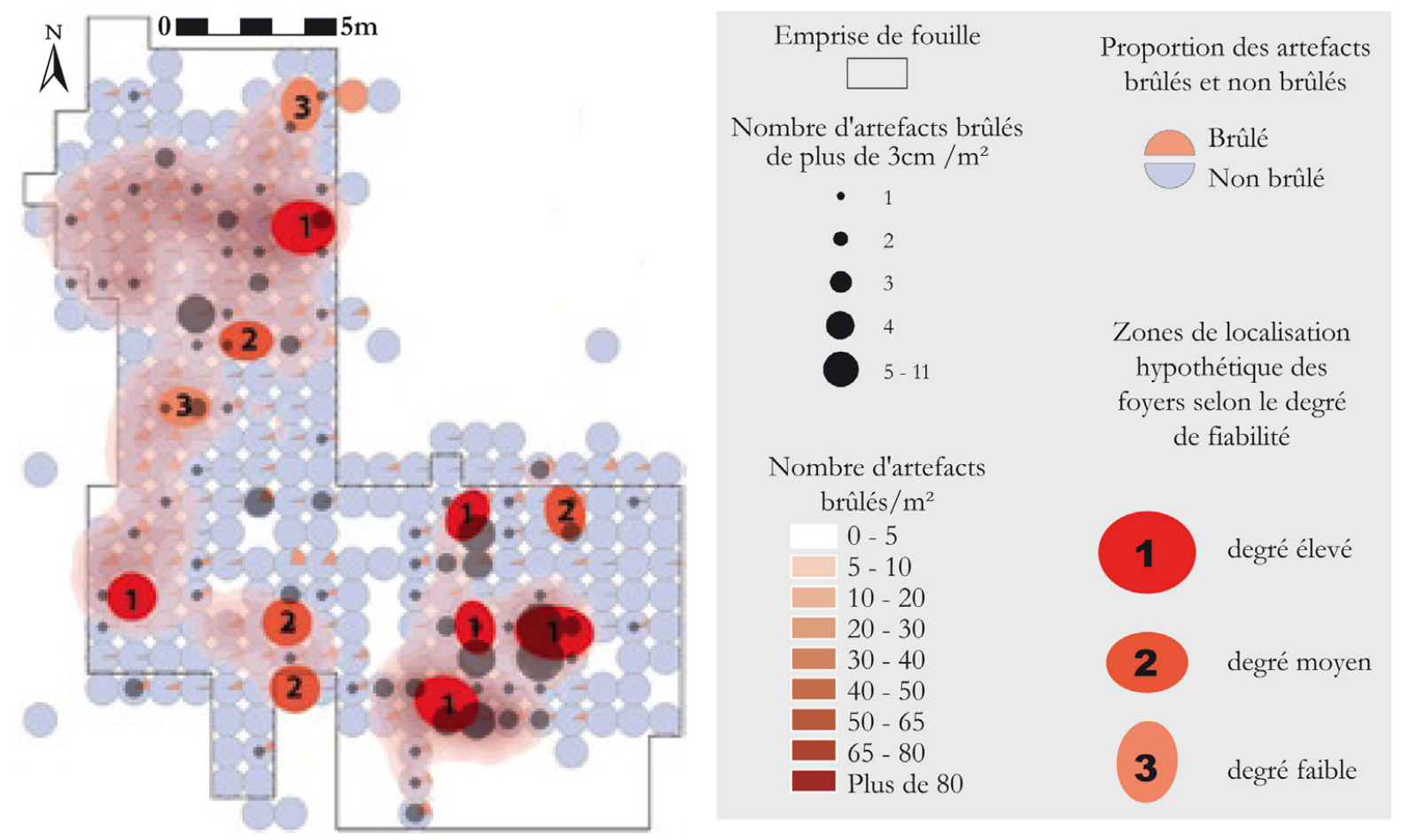

Fig. 19. Niveau N3 de Therdonne. Zones hypothétiques de localisation de foyers selon des indices de fiabilité superposées aux plans A, B et C de la Fig. 18.

Level N3 of Therdonne. Hypothetic zones of fireplaces according to reliability indexes superimposed of maps A, B and C of the Fig. 18.

\section{Premiers Néandertaliens et le feu dans le Nord de la France}

Étudier la relation entre les premiers Néandertaliens et le feu dans le Nord de la France revient à synthétiser les données acquises sur les gisements de Biache-Saint-Vaast et de Therdonne puisqu'ils demeurent à l'heure actuelle les deux uniques sites à avoir livré des traces d'utilisation de feu.

\subsection{Synthèse des résultats obtenus sur l'utilisation du feu à Biache-Saint-Vaast}

Après avoir analysé les témoins de combustion que recelaient les niveaux, des différences importantes ressortent. La première est l'inégale documentation disponible par niveau. Il est évidemment plus aisé de trancher et d'interpréter les données issues des niveaux H et IIA que de $\mathrm{D}$ et D0. La situation est claire pour le niveau $\mathrm{H}$, aucune trace de feu n'a été repérée et le recours au feu est exclu dans les limites de la zone explorée par la fouille. Le niveau IIA est sans conteste le plus riche en information. L'utilisation du feu par les Néandertaliens du niveau IIA avait été formellement affirmée. Au vu des témoins analysés, il n'y a aucun doute sur l'origine anthropique du feu. Les espèces les plus présentes dans le niveau IIA sont aussi celles ayant le plus de restes brûlés, mis à part le rhinocéros qui fait exception. L'hypothèse d'une cuisson alimentaire est avancée pour les restes d'Ursus (sp. et arctos) et de Bos primigenius. Celle de l'utilisation des bois de cervidé comme combustible est peu probable. De probables activités ayant recours à ces bois, à proximité ou en interaction directe avec le feu, peuvent être envisagées. L'usage du feu pour le niveau IIbase est bien attesté. Les mêmes phénomènes qu'en IIA s'observent : l'aurochs et l'ours dominent les assemblages et sont préférentiellement brûlés ; 
le rhinocéros bien que présent n'est pas brûlé ; le spectre des espèces brûlées est très restreint par rapport aux espèces reconnues. Les niveaux restants sont plus difficiles à cerner. Les niveaux $\mathrm{E}$ et D1 possèdent des témoins de la présence de feu mais en moindre quantité. Les silex et les os brûlés du niveau $\mathrm{E}$ et les charbons de bois et les silex brûlés du niveau D1 sont des indices d'utilisation de feu mais trop ténus pour reconstituer ses modalités d'utilisation. La question devient épineuse pour les niveaux D0 et D dont des témoins de feu sont certes présents mais la faiblesse de la documentation ne permet pas de trancher sur le statut des éléments brûlés et les modalités de l'usage du feu dans ces niveaux.

\subsection{Synthèse des résultats recueillis sur l'usage du feu à Therdonne}

Après avoir repris les observations de terrain, les résultats des expérimentations de foyer menées en parallèle de la fouille, étudié les vestiges lithiques brûlés, mené une analyse physicochimique des résidus organiques des structures de combustion, il est désormais possible de reconstituer en partie l'usage du feu par les Néandertaliens de Therdonne.

Les observations de terrain montrent clairement qu'aucun aménagement particulier n'a été détecté dans les zones noircies. L'aménagement simple de type cuvette peut éventuellement avoir été pratiqué, sans que cela soit possible de le démontrer. Les concentrations de sédiment noirci, de charbons de bois et d'éléments brûlés présentent des limites floues et n'ont pas de forme identifiable. Nous sommes donc très probablement en présence de résidus appartenant à des rejets ou des foyers affectés par divers évènements post-dépositionnels. Ces zones noircies comportent des charbons en très faible nombre et aux dimensions très réduites ainsi que de très rares fragments d'os brûlés de petites dimensions. Il est à noter la présence de 7 blocs calcaires lutétiens provenant d'au moins $3 \mathrm{~km}$, apportés sur le site, présentant des relations ambiguës avec les zones de combustion. Ces derniers sont parfois brûlés mais leur faible nombre et leur localisation dans l'occupation ne semble pas indiquer qu'ils aient pu prendre part à une structuration des foyers mais plutôt jouer un rôle dans des activités se déroulant à proximité des foyers, peut-être liées à l'alimentaire.

L'étude des silex brûlés révèle les stigmates d'une altération thermique sur plus de 3000 artefacts, soit plus de $7 \%$ de l'industrie. La majorité des silex brûlés présente les traces d'une exposition directe au feu ou à proximité immédiate du cœur de chauffe. Ces éléments témoignent d'une relation spatiale forte entre les aires de débitage et les aires de combustion.

L'analyse chimique confirme la nature anthropique des zones noircies observées sur le terrain. Les résultats des analyses chimiques menées sur les sept échantillons archéologiques convergent vers une interprétation unique des zones de combustion mises au jour. De la matière végétale a été utilisée comme combustible dont il est très probable que l'essence soit du pin (Pinus). Le pin utilisé provient certainement d'un bosquet de résineux coiffant la butte tertiaire du mont de Bourguillemont à l'époque. L'os ne semble pas utilisé comme deuxième combustible, même si la question peut rester ouverte. Quant à la destination de ces foyers, les résidus organiques préservés plaident pour de la cuisson de viande de ruminant, probablement du Boviné. Cette fonction n'était peut-être pas exclusive puisqu'un des échantillons laisse entrevoir une présence hypothétique de graisse de poisson, dont l'effective reconnaissance devra être prouvée par des analyses complémentaires.

Enfin, l'analyse spatiale des vestiges brûlés (restes osseux et surtout artefacts lithiques), des charbons de bois et des sédiments thermoaltérés démontre qu'au moins cinq foyers ont dû fonctionner sur les quelques 300 mètres carrés fouillés. L'image laissée par ces faits est celle 
d'un espace occupé régulièrement ponctué de zones de combustion circonscrites. Autour de ces zones de foyer se trouvent les zones de forte concentration lithique. Les remontages lithiques ont prouvé qu'il s'agissait d'amas de débitage (Hérisson, 2012). La localisation de ces amas de débitage semble intimement liée à la localisation des foyers. Les foyers apparaissant donc, comme il est souvent mentionné, comme des éléments centraux attracteurs, structurant l'espace ou en tout cas, nous permettant, de percevoir une répartition différentielle des activités.

\section{Conclusions}

Cette étude met en avant l'utilisation certaine du feu dans cinq des huit niveaux saaliens des gisements de Biache-Saint-Vaast (E, IIA, IIbase, D1) et de Therdonne (N3). Deux niveaux de Biache-Saint-Vaast (D, D0) présentent trop peu d'éléments pour affirmer avec certitude une utilisation du feu lors de ces occupations. À l'échelle du Nord de la France, seuls ces deux gisements recèlent des traces de combustion durant le Saalien. Ce fait atteste de la rareté des témoignages préservés de l'utilisation du feu. En effet, sept niveaux présentent des traces de feu soit plus d'un tiers des 19 niveaux pris en compte (Fig. 2). Ce ratio chute au quart des occupations présentant des traces de feu si les niveaux avec des indices incertains sont exclus. Ces ratios ne peuvent en aucun cas être utilisés tel quel pour chiffrer, modéliser, juger d'une récurrence ou d'une fréquence d'utilisation du feu par les Néandertaliens. Effectivement, la nature et l'ampleur de l'opération de fouilles, la taphonomie et le type d'occupation sont des facteurs influant fortement sur ces résultats. Tous ces éléments pris en considération, il est possible d'avancer que le feu est utilisé fréquemment sur les occupations les mieux préservées. Cependant, les problèmes taphonomiques ne peuvent expliquer à eux seuls l'absence de traces de combustion sur certains sites, également bien préservés. De ces faits observés sur les occupations néandertaliennes saaliennes du Nord de la France peuvent être tirés deux conclusions : un usage du feu non systématique mais une utilisation plutôt fréquente et plus particulièrement sur des occupations perçues comme de moyenne ou longue durée. D'un point de vue technologique, aucune trace de structure n'a pu être mis en évidence contrairement à ce qui est documenté en Normandie sur les gisements de Port Pignot et de la Roche Gélétan (Cliquet et Lautridou, 2009 ; Cliquet et al., 2003 ; Michel, 1994). Bien que les emplacements des foyers restent difficiles à cerner, la répartition des indices de combustion à Therdonne suggère une série de petits foyers, parsemés dans l'espace et fonctionnant en synergie avec les activités de débitage et probablement d'exploitation des carcasses animales. Les gisements de BiacheSaint-Vaast et Therdonne recèlent de rares et riches témoignages d'utilisation du feu par les Néandertaliens dont les résidus sont bien préservés pour leur ancienneté. Ils démontrent la maîtrise incontestable du feu par Néandertal au Saalien et son utilisation fréquente voire systématique dans certains types d'occupation de moyenne à longue durée. Ce tour d'horizon laisse entrevoir la place centrale qu'a pu occuper le feu dans le quotidien des premiers Néandertaliens du Nord de la France, dont le statut exact à leurs yeux est encore loin de nous être entièrement accessible.

\section{Remerciements}

Nous tenons à remercier Daniel Vachard pour la détermination des roches calcaires, le Centre départemental d'Archéologie du Pas-de-Calais pour l'accès aux collections de Biache-SaintVaast, ainsi que le laboratoire Archéosciences du CReAAH de Rennes pour leur accueil et l'accès 
à leur laboratoire d'analyse chimique, ainsi que le partage de leurs connaissances et de leurs protocoles pour mener à bien l'étude des foyers de Therdonne.

\section{Références}

Aldhouse-Green, S.H.R., 1995. Pontnewydd Cave, Wales; A Later Middle Pleistocene Hominid and Archaeological Site; A Review of Stratigraphy, Dating. Taphonomy and Interpretation. In: Bermudez de Castro, J.M., Arsuaga, J.L., Carbonnell, E. (Eds.), Human Evolution in Europe and the Atapuerca evidence. pp. 37-55.

Ameloot-Van der Heijden, N., 1991. Méthodes d'acquisition et signification des industries lithiques au Paléolithique moyen : l'exemple des gisements de plein air du Nord de la France. Thèse de doctoratUniversité des Sciences et Technologies de Lille Flandres Artois, Villeneuve-d'Ascq, 376.

Ameloot-Van der Heijden, N., Dupuis, C., Munaut, A.V., Puissegur, J.J., 1996. Le gisement paléolithique moyen de Salouel (Somme, France). L'Anthropologie 100, 555-573.

Antoine, P., 1989. Les terrasses quaternaires du bassin de la somme. In: Étude géologique et géomorphologique. Contribution à la connaissance du paléoenvironnement des gisements paléolithiques, Université des Sciences et Techniques de Lille Flandres-Artois, 437.

Auguste, P., 1995a. Cadres biostratigraphiques et paléoécologiques du peuplement humain dans la France septentrionale durant le Pléistocène. Thèse de doctoratIn: Apports de l'étude paléontologique des grands mammifères du gisement de Biache-Saint-Vaast (Pas-de-Calais). 5. Museum national d'Histoire naturelle, Paris, pp. 1924.

Auguste, P., 1995b. Chasse et charognage au Paléolithique moyen : l'apport du gisement de Biache-Saint-Vaast (Pas-deCalais). Bulletin de la Société Préhistorique Française 92, 155-168.

Auguste, P., 2012. L'homme et l'animal au Pléistocène en France septentrionale. Un quart de siècle de recherches paléontologiques et archéozoologiques dans le Nord de la France. Habilitation à Diriger des Recherches, 2. Université Lille 1, Villeneuve-d'Ascq, pp. 860.

Behm-Blancke, G., 1960. Altsteinzeitliche Rastplätze im Travertingebiet von Taubach, Weimar. Ehringsdorf. AltThüringen 4, 1-246.

Black, D., 1931. Evidence of the use of fire by Sinanthropus. Bulletin of the Geological Society of China 11, 107108.

Blondeau, A., Cavelier, C., Monciardini, C., 1970. Carte géologique et notice de Clermont au 1/50 000. BRGM.

Blondiau, L., Coutard, S., Ducrocq, T., Locht, J.-L., Sellier-Segard, N., 2009. Ailly-sur-Noye (Somme). « La Voirie de Guyencourt » INRAP, SRA Picardie.

Bordes, F., 1958. Compte-rendu de K P. Oakley "Fire as a palaeolithic tool and weapon" L'Anthropologie 52, 314-317.

Broca, P., 1870. L'art de faire le feu. Bulletin de la Société d'Anthropologie de Paris 5, 77-86.

Callow, P., Cornford, J.-M., 1986. La Cotte de Sainte Brelade (Jersey) 1961-1978. In: Excavations by C. B. M. Mc Burney. Geobooks, Norwich.

Clark, J.D., Harris, J.W.K., 1985. Fire and its roles in early hominid lifeways. The African Archaeological Review 3, 3-27.

Cliquet, D., 1992. Le gisement paléolithique moyen de Saint-Germain-des-Vaux/Port-Racine/Manche dans son cadre régional. Thèse de doctoratIn: Essai paléthnographique. Université de Paris I Sorbonne, 783.

Cliquet, D., Lautridou, J.P., 2009. Les occupations humaines du Pléistocène moyen de Normandie dans leur cadre environnemental. Quaternaire 20, 303-320.

Cliquet, D., Mercier, N., Valladas, H., Froget, L., Michel, D., Van Vliet-Lanoë, B., Vilgrain, G., 2003. Apport de la thermoluminescence sur silex chauffes à la chronologie de site paléolithiques de Normandie : nouvelles données et interprétations. Quaternaire 14, 51-64.

Delagnes, A., Ropars, A., 1996. Paléolithique moyen en pays de Caux (Haute-Normandie). Le Pucheuil. In: Ettouteville : deux gisements de plein air en milieu loessique. l'Homme, E.d.l.M.d.s.d.Documents d'Archéologie française, Paris.

Evershed, R.P., 1993. Biomolecular Archaeology and Lipids. World Archaeology 25, 74-93.

Green, H.S., 1983. Pontnewydd Cave and the Earlier Palaeolithic in Wales. Studia Praehistorica Belgica 3, 42.

Green, H.S., Bevins, R.E., Bull, P.A., Currant, A.P., Debenham, N.C., Embleton, C., Ivanovich, M., Livingston, H., Rae, A.M., Schwarcz, H.P., Stringer, C.B., 1989. Le site acheuléen de la grotte de Pontnewydd, Pays de Galles : géomorphologie, stratigraphie, chronologie, faune, hominidés fossiles, géologie et industrie lithique dans le contexte paléoécologique. L'Anthropologie 93, 15-52.

Heinzelin, J., Haesaerts, P.D., 1983. Un cas de débitage laminaire au Paléolithique ancien : Croix-l'Abbé à Saint-Valerysur-Somme. Gallia préhistoire 26, 189-201. 
Hérisson, D., 2012. Étude des comportements des premiers Néandertaliens du Nord de la France : les occupations saaliennes des gisements de Biache-Saint-Vaast et de Therdonne. Thèse de DoctoratUniversité de Lille Villeneuved'Ascq, 503.

Hérisson, D., Goval, E., Lefèvre, B., 2013. Éléments de réflexion sur la place de la France septentrionale en Europe du Nord-ouest durant la phase ancienne du Paléolithique moyen ». In: Depaepe, P., Goval, E., Koehler, H., Locht, J.-L. (Eds.), Les plaines du Nord-Ouest : un carrefour au Paléolithique moyen ? Société Préhistorique française, Amiens, 28-29 mars 2008.

Hérisson, D., Locht, J.L., 2013. Le niveau N3 de Therdonne : reflet de la diversité des technocomplexes du Paléolithique moyen ancien du Nord de la France. Émergence et diversité des techno-complexes du Paléolithique moyen ancien. In: Brenet, M., Bourguignon, L., Jarry, M. (Eds.), Relations entre productions de débitage et de façonnage., session C du XXVIIe Congrès Préhistorique de France. 31 mai-5 juin 2010. Société Préhistorique Française, Bordeaux - Les Eyzies.

James, S.R., 1989. Hominid use of fire in the Lower and Middle Pleistocene : a review of the evidence. Current Anthropology 30, 1-26.

Lisiecki, L.E., Raymo, M.E., 2005. A Pliocene-Pleistocene stack of 57 globally distributed benthic $\delta 180$ records. Paleoceanography 20, PA1003.

Locht, J.L., Antoine, P., Hérisson, D., Gadebois, G., Debenham, N., 2010. Une occupation de la phase ancienne du Paléolithique moyen à Therdonne (Oise). Chronostratigraphie, production de pointes Levallois et réduction des nucléus. Gallia préhistoire 52, 1-32.

Locht, J.L., Antoine, P., Swinnen, C., 1995. Le gisement paléolithique de Plachy-Buyon (Somme). Revue archéologique de Picardie 3-33.

Locht, J.-L., Guerlin, O., Antoine, P., Debenham, N., 2000. Therdonne. « Le Mont de Bourguillemont » AFAN, SRA Picardie.

Lucquin, A., 2007. Étude physico-chimique des méthodes de cuisson pré et protohistoriques. Thèse de DoctoratUniversité de Rennes I, Rennes, 422.

Mania, D., Mania, U., 2008. La stratigraphie et le Paléolithique du complexe saalien dans la région de la Saale et de l'Elbe. L'Anthropologie 112, 15-47.

March, R.J., 1995. Méthodes physiques et chimiques appliquées à l'étude des structures de combustion préhistoriques : l'approche par la chimie organique. Thèse de Nouveau Doctorat. Université de Paris I, Paris580.

March, R.J., 1996. L'Étude des structures de combustion préhistoriques : une approche interdisciplinaire. In: Bar-Yosef, O., Cavalli-Sforza, L., March, R.J., Piperno, M. (Eds.), XIII International Congress of prehistoric and protohistoric sciences, 8/14 September 1996, Colloquia 5: The Lower and Middle Paleolithic Colloquium IX, Forli (Italie).

March, R.J., Baldessari, A., Gross, E., 1989. Determinación de compuestos orgánicos en estructuras de combustión arqueológicas. In: Olive, M., Taborin, Y. (Eds.), Nature et fonction des foyers préhistoriques, Actes du colloque international de Nemours, 1987. APRAIF, Nemours.

March, R.J., Dumarçay, G., Lucquin, A., Joly, D., 2006. Les activités liées à l'utilisation du feu. Gallia Préhistoire 89-108.

Marcy, J.-L., Tuffreau, A., 1988. Le niveau D1. In: Tuffreau, A., Sommé, J. (Eds.), Le gisement paléolithique moyen de Biache-Saint-Vaast (Pas-de-Calais). Stratigraphie, environnement, études archéologiques (1ère partie) Société Préhistorique Française, pp. 263-289.

Meignen, L., Bar-Yosef, O., Goldberg, P., Weiner, S., 2000. Le feu au paléolithique moyen : recherches sur les structures de combustion et le statut des foyers. L'exemple du Proche-Orient. Paléorient 26, 9-22.

Mellars, P.A., 1976. Fire ecology, animal populations and man: a study of some ecological relationships in prehistory. Proceedings of the Prehistoric Society 42, 15-45.

Michel, D., 1994. La Roche-Gélétan. Gisement structuré du Paléolithique inférieur (Saint-Germain-des-Vaux, Manche) In: Evreux.

Munaut, A.V., 1988. Étude palynologique. In: Tuffreau, A., Sommé, J. (Eds.), Le gisement paléolithique moyen de Biache-Saint-Vaast (Pas-de-Calais). Stratigraphie, environnement, études archéologiques (1ère partie) Société Préhistorique Française, pp. 77-88.

Oakley, K.P., 1955. Fire as Palaeolithic tool and weapon. Proceedings of the Prehistoric Society 21, 36-48.

Olive, M., Taborin, Y., 1989. Nature et fonction des foyers préhistoriques. In: APRAIF. (Eds.), Actes du colloque international de Nemours 12-14 mai 1987. Mémoires du Musée de préhistoire d'Ile-de-France, Nemours.

Perlès, C., 1977. Préhistoire du feu, Paris.

Roebroeks, W., 1988. From Find Scatters to Early Hominid Behaviour. A Study of Middle Palaeolithic Riverside Settlements at Maastricht-Belvédère (The Netherlands), Leiden. .

Roebroeks, W., Villa, P., 2011. On the earliest evidence for habitual use of fire in Europe. In: Proceedings of the National Academy of Sciences. pp. 5209-5214. 
Rolland, N., 2004. Was the Emergence of Home Bases and Domestic Fire a Punctuated Event? A Review of the Middle Pleistocene Record in Eurasia. Asian perspectives 43, 248-280.

Rowlett, R.M., 2000. Fire control by Homo erectus in East Africa and Asia. Acta Anthropologica Sinica 19, 198-208 Supplément au volume.

Sandgathe, D.M., Dibble, H.L., Goldberg, P., McPherron, S.P., Turq, A., Niven, L., Hodgkins, J., 2011. Timing of the appearance of habitual fire use. Proceedings of the National Academy of Sciences 108, E298.

Schäfer, D., Jäger, K.-D., 1984. Verkohlte Steinkerne der Kornelkirsche (Cornus Mas L.) aus dem Paläolithikum des oberen Travertins von Weimar-Ehringsdorf. Alt-Thüringen 20, 15-22.

Schiegl, S., Goldberg, P., Bar-Yosef, O., 1996. Ash deposits in Hayonim and Kebara Caves, Israel: macroscopic, microscopic and mineralogical observations, and their archaeological implications. Journal of Archaeological Science 23, 763-781.

Sommé, J., 1988. Géomorphologie et stratigraphie. In: Tuffreau, A., Sommé, J. (Eds.), Le gisement paléolithique moyen de Biache-Saint-Vaast (Pas-de-Calais). Stratigraphie, environnement, études archéologiques (1ère partie) Société Préhistorique Française, pp. 27-45.

Stapert, D., 2007. Youngsters knapping flint near the campfire: an alternative view of Site K at Maastricht-Belvédère (The Netherlands). "A refitter's paradise" Archäologisches Korrespondenzblatt 37, 19-35.

Steiner, W., 1975. Zur stratigraphischen Stellung des Travertinprofils von Ehringsdorf bei Weimar. Alt-Thüringen 12, 716.

Théry-Parisot, I., 2001. L'économie des combustibles au Paléolithique. CEPAM, Paris.

Théry-Parisot, I., Chabal, L., Costamagno, S., 2010.In: Taphonomie de la combustion des résidus organiques en contexte archéologiques. Actes de la table ronde, Valbonne, 27-29 mai 2008 Palethnologie.

Tuffreau, A., 1978. Les fouilles du gisement paléolithique de Biache-Saint-Vaast (Pas-de-Calais) : années 1976 et 1977 premiers résultats. Bulletin de l'Association Française pour l'Étude du Quaternaire 15, 46-55.

Tuffreau, A., 1987. Le Paléolithique inférieur et moyen du Nord de la France (Nord Pas-de-Calais, Picardie) dans son cadre stratigraphique. Thèse de doctorat d'État. Université des Sciences et Techniques de Lille Flandre Artois, Lille 609.

Tuffreau, A., 1988a. Les habitats du Paléolithique inférieur et moyen dans le Nord de la France (Nord, Pas-de-Calais Somme). Revue archéologique de Picardie 91-104.

Tuffreau, A., 1988b. Stratigraphie de la séquence archéologique. In: Tuffreau, A., Sommé, J. (Eds.), Le gisement paléolithique moyen de Biache-Saint-Vaast (Pas-de-Calais). Stratigraphie, environnement, études archéologiques (1ère partie) Société Préhistorique Française, pp. 123-131.

Tuffreau, A., 2012. Les premières indutries lithiques dans le Nord de la France, reflet des premiers peuplements dans une région de haute latitude : état des connaissances. Les Nouvelles de l'Archéologie 36-41.

Tuffreau, A., Bouchet, J.-P., 1985. Le gisement acheuléen de la Vallée du Muid à Gouzeaucourt (Nord). Bulletin de la Société préhistorique française 82, 291-306.

Tuffreau, A., Sommé, J., 1988a. Le gisement paléolithique moyen de Biache-Saint-Vaast (Pas-de-Calais). In: Stratigraphie, environnement études archéologiques (1ère partie). Mémoires de la Société Préhistorique Française. Société Préhistorique Française.

Tuffreau, A., Sommé, J., 1988b. Le gisement paléolithique moyen de Biache-Saint-Vaast (Pas-de-Calais). In: Stratigraphie, environnementétudes archéologiques (1ère partie). Société Préhistorique Française. 